\title{
La discrimination et ses formes dans La tresse de Colombani
}

\author{
par \\ Abir Chéhata* \\ Dr_abirchehata@du.edu.eg
}

\section{Résumé}

Dans son récit La tresse, Colombani raconte l'histoire de tro is femmes, trois vies dans trois pays différents. Le premier, l'Inde, où vit Smita,dans le village de Badlapur. Elle est une malheureuse intouchable, mariée à un chasseur de rats, qui, comme le cas de tous les intouchables de son village, la caste la plus pauvre de ce pays, accomplit des tâches les plus indignes en nettoyant les toilettes des castes plus nobles comme le faisait sa mère.

Pour Smita, une chose est sûre, celle de voir sa fille de six ans à peine, Lalita, échapper à sa condition minable en entrant à l'école.

Le deuxième, l'Italie, où vit Giulia, en Sicile, plus exactement à Palerme, elle travaille dans l'atelier de traitement de cheveux, dirigé par son père. Victime d'un accident, il entre dans un coma profond. Giulia découvre brusquement que l'entreprise familiale est au bord de la ruine. Il lui faut donc trouver une solution pour sauver cette situation.

Et le troisième, le Canada, où vit Sarah, à Montréal, plus précisément, une a vocate renommée ayant trois enfants. Elle va être promue à la tête de son cabinet quand le médecin lui apprend qu'elle est atteinte d'un cancer.

Colombani veut bien appuyer sur les évènements marquants dans chaque histoire. De même, elle désire avouer son point de vue contre la

* Maître de conférences - Département de langue et de littérature françaises -Université de Damiette 
discrimination car chaque évènement se rapporte à une des conséquences négatives de la société.

Mots clés : Colombani ,La tresse, La discrimination et ses formes: Discrimination raciale, discrimination de situation de familie, discrimination de l'etat de sante.

\section{Introduction}

Laetitia Colombani, née en 1976 à Bordeaux, est une réalisatrice, scénariste et écrivaine française.

Après deux années de classe préparatoire cinésup à Nantes, elle entre à l'École Nationale supérieure Louis Lumière. Elle obtient son diplôme en 1998.

Laetitia Colombani écrit et réalise des courts - métrages, puis deux longs - métrages : À la folie ... pas du tout (2002) avec Audrey Tautou, Samuel le Bihan et Isabelle Carré, remportant le prix Sopadin junior du Meilleur Scénario, puis Mes stars et moi (2008) avec Kad Merad et Catherine Deneuve.

Elle travaille également pour la scène en coécrivant la comédie musicale Résiste en 2015 d'après les chansons de France Gall crées par Michel Berger (Palais des sports de Paris et tournée dans toute la France)

Aussi, Colombani est comédienne à la télévision et au cinéma, dans une douzaine de longs métrages dont cloclo de Florent Emilio Siri, paru en 2012, et fête de famille, de Cédric Kahn en 2019.

Son premier roman" La tresse " paraît chez Grasset en mai 2017. Le roman remporte plusieurs prix dont le $40^{\circ}$ prix Relay des Voyageurs Lecteurs 2017, le Trophée littéraire 2017 des Femmes de l'Économie et le Globe de Cristal 2018 du Meilleur Roman, le Prix de l'Office Central des

(La discrimination et ses formes ...) Dr. Abir Chéhata 
bibliothèques, le Prix des Zonta clubs de France et le Prix de la FNABEH (Fédération Nationale des Associations de Bibliothèques en établissements Hospitaliers).

Ce roman s'est vendu à près d'un million d'exemplaire en France et a été traduit dans 35 langues. Il a été décliné en album enfants, aussi en album jeunesse, la tresse ou le voyage de Lalita.

$\ll$ On ne sait pas $\ll$ pourquoi, certains livres, avant même leur parution, avant même qu'ils aient été lus (..) s'annoncent comme des phénomènes, sont de la graine de best - sellers. C'est le cas avec ce premier roman <<la tresse $>>$, déjà vendu à une dizaine de pays, dont $l^{\prime}$ ' Allemagne qui s'est complètement entichée du manuscrit.>> (1)

Colombani publie en 2019, son deuxième roman, "les victorieuses ", qui comme le premier, a eu un grand succès public.

Notre choix du corpus sera porté sur La tresse de Colombani or $\ll$ Tresse n.f. Assemblage de trois mèches, de trois brins entrelacés $>>(2)$

D'après cette définition qui se trouve au début du roman, nous remarquons que

le titre est significatif de sa structure. Dans son récit, Colombani raconte l'histoire de trois femmes, trois vies dans trois pays différents. Le premier, l'Inde, où vit Smita, en Uttar, Pradesh, dans le village de Badlapur. Elle est une malheureuse intouchable, mariée à un chasseur de rats, qui, 
comme le cas de tous les intouchables de son village, la caste la plus pauvre et la plus négligée, de ce pays, accomplit des tâches les plus indignes en nettoyant à mains nues les toilettes des castes plus nobles comme le faisait sa mère.

Smita n'arrive guère à se défaire de cette odeur de merde qui lui fixe à la peau, imprégnant chaque partie de son corps. Mais pour elle, une chose est sûre, celle de voir sa fille de six ans à peine, Lalita, échapper à sa condition minable en entrant à l'école et en lui offrant une éducation qu'elle n'a pas obtenue.

Le deuxième, l'Italie, où vit Giulia, en Sicile, plus exactement à Palerme, elle travaille dans l'atelier de traitement de cheveux, dirigé par son père. Victime d'un accident, il entre dans un coma profond. Giulia découvre brusquement que l'entreprise familiale est au bord de la ruine et qu'elle ne peut durer qu'un mois de plus.

Il lui faut donc trouver une solution pour sauver cette situation. Et le troisième, le Canada, où vit Sarah, à Montréal, plus précisément, une avocate renommée, si active et compétitive, habitant dans un quartier aisé, ayant trois enfants qu'elle éduque seule avec l'aide de Ron, qui remplit le rôle de nounou à domicile et l'appuyant dans la plupart des tâches domestiques, va être promue à la tête de son cabinet quand le médecin lui apprend qu'elle est atteinte d'un cancer. Sarah, cette femme douée, a beau vouloir dissimuler sa maladie à son patron et à ses collègues et poursuivre à gagner ses dossiers mais le secret finira par se divulguer.

Trois femmes, sans jamais se connaître, sans jamais se croiser vont se trouver liées sans le savoir parce qu'elles ont de plus personnel et de plus particulier. 
Selon Genette, le récit est définit comme étant $: \ll$ la représentation d'un événement ou d'une suite d'événements, réels ou fictifs, par le moyen du langage,et plus particulièrement du langage écrit.>> (3) Ce même récit est jugé par Genette comme étant une base sur laquelle repose la narration $: \ll$ Tout récit comporte[..] des représentations d'actions et d'événements, qui constituent la narration proprement dite >> (4)

Dans une interview, Pierre Krause a demandé à Colombani à propos de son roman en disant :

$<<$ (...) Qu'est - ce qui réunit ces trois

femmes et qu'est -ce qui vous intéressez dans leurs parcours respectifs?

Je voulais construire mon récit autour de trois personnages féminins, qui, en apparence ,n'ont rien en commun. (....) mais chacune, à sa manière, est enfermée, cantonnée à un rôle qu'on lui a assigné. Chacune endure une forme de discrimination. $>>(5)$

Smita, Giulia et Sarah refusent la discrimination qui leur est soumise et décident de se battre. Leurs histoires trament une tresse de résiliation, de dépendance et d'espoir.

Colombani déclare :

$\ll$ Je dédie mon travail à [...]celles qui aiment, enfantent, espèrent, tombent et se révèlent, mille fois, qui plient mais ne succombent pas.>> (6)

Nous allons nous concentrer sur le thème de la discrimination sous ses diverses formes. Le mot discrimination provient $\mathrm{du}$ mot latin discriminis, qui exprime 
$<$ Séparation $>>$.

Ce mot discrimination est admis dans le langage courant surtout dans celui des sciences sociales avec un sens plus limité. Au sens courant, la discrimination est un choix ou un acte ayant pour effet de traiter de manière inégale et adverse un ou maintes personnes. Plus précisément, il s'agit de discerner un groupe social des autres en fonction de caractères extérieurs (éducation, logement) ou intérieurs (sexe, origine, ethnique) afin de pouvoir lui faire servir pour un traitement particulier généralement négatif.

Toute discrimination est illégale, blâmable et critiquable. Elle porte atteinte à l'honneur ou à la probité absolue des personnes.

Vanter des inégalités, insulter, menacer, à raison d'une race, d'une religion, ou d'une situation de santé particulière sont des attitudes discriminantes.

\section{1-Définition légale de la discrimination :}

Le code pénal, dans sa section $<<$ Des discriminations $>>$ du chapitre voué aux $<$ atteintes à la dignité de la personne $>$, distingue de nombreux types de discriminations

$<<$ L'article 225 - 1-Modifié par LOI $n^{\circ}$

2016- 1547 du 18 novembre 2016-art.

86- définit une liste de critères qui entrent dans la constitution d'une discrimination :

$<$ constitue une discrimination toute distinction opérée entre les personnes physiques sur le fondement de leur origine, de leur sexe, de leur situation de famille, (...) de leur patronyme, (...) de leur état de santé, de leur perte d'autonomie, (...) de leur appartenance 
ou de leur non - appartenance, vraie ou supposée, à une ethnie, une Nation, une prétendue race ou une religion déterminée. >> (7)

Cette même définition est signalée par la romancière Colombani aux pages 159 -160 du roman en y ajoutant encore que ce terme est parfois allié à celui de $\quad<<$ stigmate >> tel que le sociologue Erving Goffman le fixe

$\ll$ Attribut qui rend l'individu différent de la catégorie dans laquelle on voudrait le classer. $>>(8)$

Un individu qui en est donc attristé, chagriné est donc stigmatisé, qui s'opposant aux autres que Goffman nomme les normaux.

\section{A. Discrimination raciale}

Dans l'histoire de Smita, nous remarquons la présence de deux castes dans la société indienne. Il y a les castes supérieures représentant les Jatts, les Brahmanes et les castes inférieures comme celle des Intouchables.

$<$ Comme Smita, ils sont des millions à vivre en dehors des villages, de la société, à la périphérie de l'humanité. $>>{ }^{(9)}$

D'un côté, les castes supérieures figurent au sommet de l'échelle sociale, d'un autre, les Intouchables désignent le bas fond de cette échelle. Cette hiérarchie sociale évoque la discrimination faite à l'égard des Intouchables. Une discrimination visée d'une caste sur une autre caste. Aussi

$<<$ le racisme est l'attitude spontanée des dominants, $<$ une habitude mentale liée à une structure sociale > (10), un 
ensemble, de convictions et d'assurances propres à ceux qui occupent une position dominante : $\ll$ Plus les gens sont en position de dominants, et plus ils ont une tendance spontanée au racisme de classe >>. (11)

Dans le dictionnaire Larousse, la discrimination est le $<<$ fait de distinguer et de traiter différemment (le plus souvent plus mal) quelqu'un ou un groupe par rapport au reste de la collectivité ou par rapport à une autre personne >>.$^{(12)}$

Comme le montre Colombani

$<$ Smita entre dans les maisons par la porte arrière qui lui est réservée, elle ne doit pas croiser les habitants, encore moins leur parler. Elle n'est pas seulement intouchable, elle doit être invisible. $>>(13)$

Smita travaille comme $<$ Scavenger $>>$ terme anglais signifiant $<<$ extracteur $>>$, elle ramasse les merdes humaines à mains nues toute la journée.

$\ll$ Elle lave son visage et ses pieds à l'eau rapportée la veille du puits, celui qui leur est réservé. Pas question de toucher à l'autre, celui des castes supérieures, pourtant proche et plus accessible.>> (14)

À sept heures, Smita commence sa tournée en prenant son panier et sa balayette en jonc. Elle n'a pas de temps à perdre parce qu'elle doit vider vingt maisons, Smita doit souvent faire son travail à temps pour ne pas être punie par les habitants des castes supérieures chez lesquelles elle travaille. Elle marche les yeux baissés, le visage caché sous un foulard. Dans quelques villages, les Dalits signalent leur présence en 
mettant une plume de corbeau. Dans d'autres, ils marchent pieds nus

$<<$ tous connaissent l'histoire de cet

Intouchable, lapidé pour le seul fait

d'avoir porté des sandales. $>>$ (15)

Ce métier médiocre se transmet de mère en fille depuis des générations

$\ll$ [Smita] reçoit en guise de salaire des restes de nourriture, parfois des vieux vêtements, qu'on lui jette à même le sol. Pas toucher, pas regarder. >>(16)

Le théoricien Greimas a rangé les personnages sur la base de leur fonctionnalité, de leur faire. Ils sont assemblés dans des catégories communes et perçues tels que des forces agissantes (appelés actants), primordiales à toute intrigue.

Quant à Hamon, il a analysé le personnage non pas à travers ce qu'il fait, mais comme un être de papier doté d'un nom, d'un portrait comprenant des traits physiques et moraux. Or le portrait du personnage peut se rapporter au corps, à l'habit (qui révèle l'origine sociale et culturelle du personnage), la psychologie qui

donne l'idée de la vie intérieure d' un personnage ainsi que sa biographie en faisant rappel à son passé, elle permet ainsi de fortifier le vraisemblable psychologique du personnage.

Son mari Nagarajan part tôt, comme son père, dès le lever du soleil pour chasser les rats qui rongent les récoltes des champs des Jatts en creusant de petits trous.

$\ll C^{\prime}$ est une tradition ancestrale, un savoir - faire(..) d'attraper les rats à mains nues.>> (17) 
Les rats grillés ressemblent au poulet. Ce sont les poulets des pauvres, ceux des Dalits. Nagarajan aime manger les rats entiers ne laissant que la queue. Smita préfère enlever la peau.

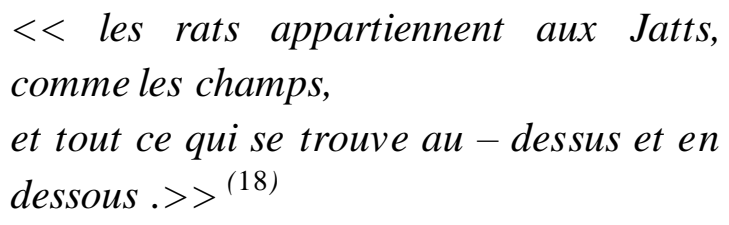

Les travaux durs et modestes de ces castes inférieures ne leur rapportent pas de bénéfices.

Dans un entretien, Pierre Krause a questionné Colombani à propos de l' héroïne indienne en disant

$<<$ (...) Sa vie, très difficile dans le roman, reflète - $t$ - elle - celle que vivent aujourd'hui des millions d'Intouchables en Inde? Leurs conditions de vie sont elles toujours aussi difficiles au XXI-ème siècle?

(...) Je suis moi-même allée en Inde il y a quelques années, et ai visionné des dizaines d'heures de documentaire sur la condition des Intouchables là- bas-et des femmes en particulier. Ce qu'ils endurent est inimaginable. Pour eux rien n'a changé au fil des siècles, le système des castes les maintient dans très grande précarité. Certes le gouvernement fixe des quotas de places réservées dans l'administration et les universités, mais malgré cela, la discrimination perdure.>> (19) 
Smita et son mari habitent un lieu enfermé, étroit et sombre qui leur sert à la fois de cuisine, de chambre et de temple. Quant aux Jatts, ceux-ci habitent des maisons qui se situent au centre du village. Or, la possession d'une de ces maisons par les Intouchables est presque impossible.

$<$ le racisme se diffuse toujours de haut, en bas, des dominants vers les dominés, qu'il contamine pour ainsi dire. Le< virus > affecte préférentiellement les dominants, et descend vers les innocents pas sa nature. (...) le racisme est défini comme une forme de $<$ déni d'humanités> (20)

La situation reste de plus en plus pire parce que à Badlapur $\ll$ Partout le sol est souillé, les rivières, les fleuves, les champs, pollués par des tonnes de déjections. Les maladies s'y propagent comme une étincelle sur de la poudre. >> (21)

Aussi

$<<$ les femmes sont obligées d'attendre la tombée de la nuit pour aller dans les champs, s'exposant à de multiples agressions.>>(22)

Même l'école, ce lieu d'instruction est interdit aux Dalits. Ils n'ont pas le droit d'y entrer.

$<<$ À l'école, Smita n'y a jamais mis les pieds. Ici à Badlapur, les gens comme elle n'y vont pas. Smita est une Dalit. 
Intouchable. De ceux que Gandhi appelait les enfants de Dieu.

Hors caste, hors système, hors tout.>> (23)

Mais Smita prend une décision celle d'envoyer sa petite à l'école pour savoir lire et écrire. Elle refuse d'emmener Lalita avec elle durant sa tournée. Smita ne lui montre jamais les gestes des videurs de toilettes, elle déteste de voir sa fille vomir dans le fossé comme sa mère avant elle, non elle refuse absolument.

$\ll$ L'évocation d'une vie intérieure est une technique connue de l'illusion de personne. La référence aux pensées, sentiments, passions, angoisses ou désirs d'un personnage, donne une impression de $<<$ richesse psychique $>>$. (...) Aucun personnage ne semble plus vivant que ceux dont le texte éclaire l'intériorité.>> (24)

Devant l' insistance de Smita, Nagarajan finit par accepter.

<Il connaît sa femme; sa volonté est puissante. Cette petite Dalit à la peau brune qu'il a épousée il y a dix ans est plus forte que lui,il le sait. Alors il finit par céder. Soit. Il ira à l'école du village, il parlera au Brahmane. >> (25)

Le Brahmane accepte Lalita à l'école, il prend la boîte dans laquelle Smita met toutes les économies pendant des mois et des mois pour cette raison.

$<$ Smita le sait, tout fonctionne ainsi.

L'argent est force de persuasion ici.>> (26)

(La discrimination et ses formes ...) Dr. Abir Chéhata 
Smita entre dans la cahute après un long jour de travail, elle trouve Lalita muette, assise dans un coin, son regard fixé au sol. Son sari est déchiré, ce sari neuf que Smita reste à c oudre des nuits après des nuits, refusant de dormir pour être prêt à temps. Ce sari faisant sa hauteur $\ll$ est déchiré, abîmé, souillé !>> (27)

$\mathrm{Au}$ premier abord, Smita croit que sa fille est désobéissante c'est pourquoi le maître ne veut pas la prendre. Cela ne veut dire que retourner aux latrines, à la merde des autres. À ce panier affreux dont elle veut tant l'en éloigner. Fou de rage, Smita se met à frapper sa fille qui, à son tour, essaye de protéger son visage. Nagarajan rentre des champs, il se précipite en entendant des cris dans la cour. Il se dresse entre sa femme et sa fille, il arrive à l'écarter en prenant Lalita dans ses bras.

$$
\begin{aligned}
& <\text { Il découvre les marques de coups } \\
& \text { dans son dos, les zébrures sur la peau } \\
& \text { fendue. Il serre l'enfant contre lui. >> (28) }
\end{aligned}
$$

Après un moment de silence, Lalita finit par dire une phrase < < qui vient les gifler tous deux: Il voulait que je balaye la classe. (..)Il voulait que je balaye devant les autres. J'ai dit non.>> (29)

Ainsi

$$
\begin{aligned}
& \ll \text { un tel usage élargi du mot racisme } \\
& \text { renvoie indistinctement à des attitudes, à } \\
& \text { des convictions idéologiques implicites, à } \\
& \text { des systèmes idéologiques et à des } \\
& \text { pratiques. }>>(30)
\end{aligned}
$$

À l'école, là où faudrait prédominer une égalité entre toutes les élèves, Lalita, l'une d'entre elles, est traitée d'une manière différente et négative. Cette discrimination n'est donc qu'une 
violation du principe d'égalité. Le fait d'être humilié signifie que la personne est témoin d'un acte de violence.

$\ll$ cette discrimination devient un harcèlement. (...) On dit que l'exclusion va de pair avec la discrimination, car une personne qu'on traite différemment est mise en dehors du groupe.>> (31)

En outre $<<$ (...) il n'y a rien de plus difficile à comprendre qu'une différence de traitement qui ne repose sur aucune base logique, c'est-à-dire sur aucun argument de poids et objectif. >> (32)

À cet instant, nous confirmons que Smita déteste de plus en plus la condition dans laquelle elle vit, elle et sa famille. Mais elle est quand même satisfaite de sa fille le jour où elle a su braver le maitre de l'école qui l'ordonne de balayer devant les autres camarades.

$\ll$ Lalita ne s'est pas laissée faire. Elle a dit non. À cette pensée, Smita se sent fière de sa fille. Cette enfant de six ans, à peine plus haute qu'un tabouret, a regardé le Brahmane dans les yeux et lui a dit: non. Il l'a attrapée, l'a frappée avec sa baguette en jonc, au milieu de la classe ,...>> (33)

De même

< On parle d'inégalité de traitement lorsqu'un individu ou un groupe d'individus fait l'objet d'un traitement moins avantageux qu'un autre individu ou qu'un autre groupe d'individus, alors qu'il 
se trouve dans une situation identique au comparable.>> (34)

Cette inégalité de traitement est jugée comme discriminatoire lorsque le traitement moins avantageux est droitement lié à l'appartenance à un groupe distinctif ou à une caractéristique individuelle particulière et que l'on ne peut pas espérer d'une personne qu'elle les corrige. Ces caractéristiques se discernent par le fait qu'elles sont péniblement changeables

$\ll$ le racisme est une idéologie dont le noyau dur est constitué par l'affirmation d'une inégalité, fondée sur des différences de nature, entre les groupes humains (races), affirmation qui implique des pratiques d'exclusion, de discrimination, de persécution ou d'extermination, que préparent et / ou accompagnent des attitudes de haine et de mépris (de $\left\langle<l^{\prime}\right.$ autre $\rangle$. $^{(35)}$

Sans cesse, Smita se met à répéter

$<<(. .$.$) elle mange peut-être du rat mais$
elle a plus de force que tous ces
Brahmanes et ces Jatts réunis, ils ne l'ont
pas domptée, pas écrasée. Ils l'ont rouée
de coups, zébrée de cicatrices mais elle
est là, au - dedans d'elle - même.
Intacte.>> (36)

Cette nuit, Smita n'arrive pas à dormir, elle ne trouve plus le sommeil. Colombani décrit la nuit comme un temps d'ennui et d'insomnie

$\ll$ Ces nuits ne lui offrent plus rien qu'un abîme sans fond, 
des rêves aussi noirs que la fange qu'elle nettoie.>> (37)

Après la scène de frappement de sa fille Lalita, Smita décide de ne plus se taire, de se révolter et de s'enfuir du village avec sa fille

$<$ [elle] préférerait mourir que de l'envoyer à nouveau à l'école; Lalita n'y mettra plus les pieds. Elle maudit cette société qui écrase ses faibles, ses femmes, ses enfants, tous ceux qu'elle devrait protéger. ${ }^{(38)}$

L'unique solution se réduit à

$\ll$ Partir, fuir cet endroit. C'est la seule issue. $>>(39)$

Ainsi

$<(.$.$) Le vouloir transforme le$ personnage en un actant-sujetorienté vers un but: <<Le mentionner(..) dans un roman, c'est donc référer le lecteur à une permanence, à la globalité d'une structure narrative, c'est donc construire un système d'appels et de rappels, une cohérence, celle d'une $<<$ personne $>>$ et celle d'un récit, en référant un sujet à son projet, des causes à des conséquences ou des conséquences à une cause >>. ${ }^{(40)}$

Smita décide de fuir, de prendre un billet sans retour $\ll$ un aller simple pour une vie meilleure. Plus jamais l'enfer du petit village de Badlapur. Smita se l'est promis .> (41) 
Nous sentons de l'admiration et de l'estime envers le personnage de Smita. Or, malgré sa pauvreté, elle arrive à prendre une décision tranchante.

$<$ La sympathie ou l'antipathie pour un personnage (...) dépendent essentiellement des caractéristiques psychologiques ou morales (ou physiques!) que lui prête l'auteur, des conduites et des discours qu'il lui attribue , et fort peu des techniques du récit où il figure $>{ }^{(42)}$

Contrairement à l'avis de Smita, son mari Nagarajan est soumis à sa fatalité ; il préfère accepter son destin en disant :

$<<(. .$.$) passer le balai, après tout, ce$ n'est pas si terrible,un coup de balai, ça fait moins mal qu'un coup de baguette en jonc ... $>>{ }^{(43)}$

De surcroît, Nagarajan essaye de retenir sa femme en la faisant bien comprendre la situation.

$\ll$ Lalita doit accepter les brimades, si elle veut retourner à l'école. C'est à ce prix qu'elle apprendra à lire et à écrire. C'est ainsi dans leur monde, on ne sort pas impunément de sa caste. Tout se paye ici.>> (44)

Nagarajan croit que sa femme a perdu la tête. Il essaye alors de lui rappeler l'histoire terrible de la fille d'un de leurs voisins, une Dalit comme elle, avait pris décision de partir étudier en ville. Les Jatts l'ont surpris lors qu'elle s'évadait à travers la campagne. 
Ils l'ont amené dans un champ abandonné et l'ont violée à huit, pendant deux jours

$<<(. .$.$) la discrimination peut (...)$
prendre des formes indirectes, servant
ainsi de socle à la violation d'autres
droits humains comme la privation des
libertés, des traitements cruels et
dégradants, des crimes contre l'humanité.
$>>(45)$

Les parents de la fille violée ont porté plainte auprès du Panchayat. Le conseil de village, qui incarne l'autorité. Certainement, il est aux mains des Jatts. Chaque décision du conseil dispose la force de la loi même si elle contrarie la constitution indienne.

$\ll$ Le conseil a proposé quelques billets à la famille pour la dédommager, en échange du retrait de la plainte, mais la jeune femme a refusé l'argent de la honte. Son père a tenté de la soutenir, puis a fini par [se donner]la mort, abandonnant sa famille sans ressources, (...) [sa femme] et ses enfants ont été bannis du village, (...) Ils ont fini dans le dénuement le plus total, au bord d'une route, dans un fossé.>> (46)

Effectivement,

$\ll$, (..) le droit communautaire est audessus des lois nationales dans la hiérarchie des sources de droit. Il est donc possible de mettre en cause ces lois 
comme discriminatoires au regard de ce droit.>> (47)

Ainsi

$<$ Abolir la loi, certes non; mais il est permis de souhaiter (..) qu'elle tombe peu à peu en désuétude. (...) une attaque (..)faite à un seul constitue en réalité une menace pour l'ensemble de la société.>> (48)

Les arguments effrayants de Nagarajan n'ont aucune importance face à l'entêtement de sa femme Smita. Elle et sa fille rejoindront d'abord la ville sacrée Varanasi où elles prendront un train pour traverser l'Inde jusqu'à Chennai : des cousins de sa mère vivent là - bas, ils les soutiendront. Cette ville se situe au bord de la mer, on raconte qu'un homme a aménagé une communauté de pêcheurs pour les scavengers, les gens tels son cas.

« Il existe aussi des écoles pour les enfants Dalits. Lalita saura lire et écrire. Ils trouveront du travail. Ils n'auront plus à manger du rat. >> (49)

Notons encore

$\ll$ Toutes les normes d'égalité ne bénéficient pas de la reconnaissance accordée aux Droits de l'homme ou de l'autorité d'une constitution. (...) différents groupes cherchent à faire prévaloir leur norme d'égalité. Le concept de discrimination devient alors un outil dans ce but. Cependant, davantage qu'une lutte contre la 
discrimination, il s'agit alors d'une lutte pour l'égalité. >> (50)

Après avoir pris la décision de fuir, Smita prie pour elle et sa fille. De même, elle pense à tous les Intouchables qui vivent dans la même condition inhumaine qu'elle. Elle souhaite les voir se rebeller, se révolter eux aussi.

$<<(. .$.$) elle n'était plus seule, comme si$ des millions d'Intouchables étaient agenouillés là, devant le petit autel, et priaient avec elle.>> (51)

Un long voyage à parcourir à pied, à vélo, en bus, en train jusqu' à Chennai. Un voyage fatiguant inquiétant, à l'issue inconnue. Alors. Smita promet Vishnou si elle et sa fille réussissent à fuir., si la femme du Brahmane n'observe rien, si les Jatts me les happent pas, si elles arrivent jusqu' à Varanasi et si enfin, elles atteignent, le sud, en vie, elles iront donc lui rendre hommage au temple Tirupati. Ce grand lieu de pèlerinage, sur le montage de Tirumala, où des millions, chaque année viennent faire des offrandes au seigneur de la montagne.

\begin{abstract}
$<$ Selon [Greimas et Larivaille], tout récit serait fondé sur la super - structure suivante, que l'on appelle aussi schéma canonique du récit ou schéma quinaire, en raison de ses cinq grandes <<étapes>> : Le récit se définirait ainsi comme transformation d'un état en un autre état. Cette transformation est constituée d'un élément qui enclenche le procès de transformation, de la dynamique qui l'effectue (ou non) et d'un
\end{abstract}


autre élément qui clôt le procès de transformation. >> (52)

\section{TRANSFORMATION}

État initial

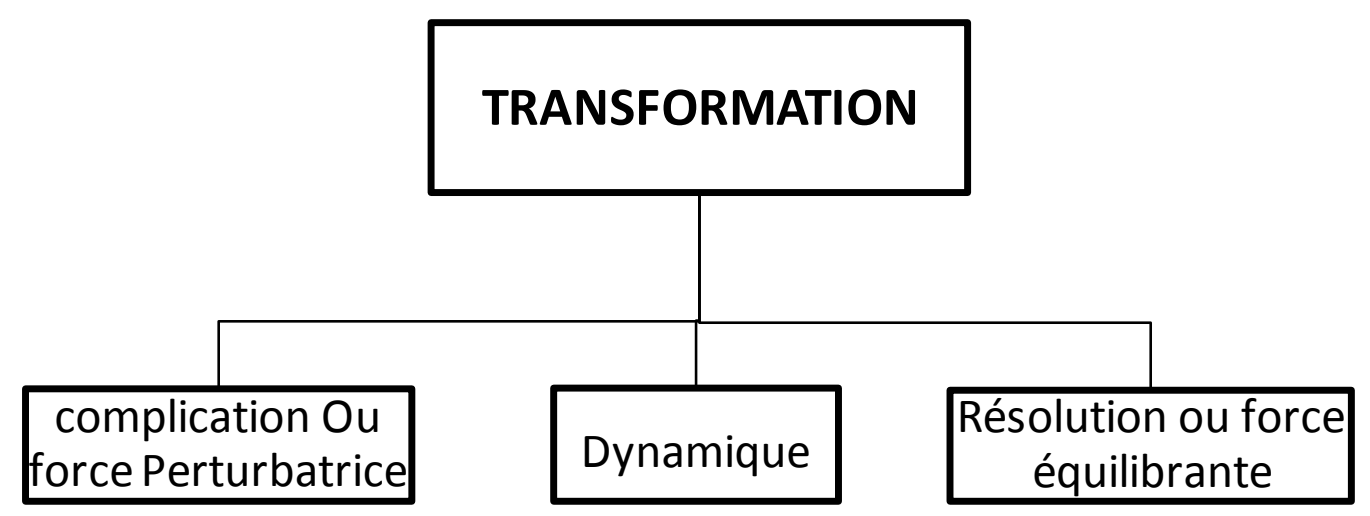

L'état de Smita peut durer éternellement. Pour qu'il y ait histoire (c'est-à-dire un $<<$ faire $>>$, des actions ...), il faut que quelque chose ou quelqu'un perturbe cet état en entrainant une série d'événements tel l'acte du Brahmane d'humilier sa fille devant ses camarades, cet état pourrait demeurer inchangé si Smita ne décide pas de partir pour la délivrer. Cette décision va entraîner toutes les luttes, les attaques (la dynamique). Mais après tout, une progression d'actions déclenche un nouvel élément (la résolution) qui va donc s'introduire pour achever la transformation. À partir de là, un nouvel état se fonde.

\section{B. Discrimination de situation de famille :}

Dans l'histoire de Giulia, nous trouvons deux classes. La première, la classe ouvrière représentée par les femmes qui travaillent dans l'atelier des perruques

(La discrimination et ses formes ...) Dr. Abir Chéhata 
$<<(.$.$) observer leurs mains en train de$ s'activer telle une armée de fourmis. (...) jeter les cheveux sur les cardes, ces grands peignes carrés, pour les démêler, puis les laver dans la baignoire fixée sur des tréteaux ...>> (53)

Et la deuxième la classe patronale incarnée par Pietro Lanfredi :

$\ll$ Symbole de la génération, de la
possession (...) Il est une représentation
de toute figure d'autorité chef, patron,
professeur... $>>(54)$

Cette dernière classe montre par excellence l'une des formes de discrimination bâtie en 1926 par l'arrière - grand - père de Giulia, l'atelier des perruques représente le dernier de ce genre à Palerme dans lequel travaille une dizaine d'ouvrières spécialisées. Ces ouvrières démêlent, lavent et traitent des mèches envoyées après en Italie ainsi que dans toute l'Europe.

Pierre Lanfredi, le chef de ce secteur installe l'atelier de son père dans un ancien cinéma dont il rachète les murs. Aujourd'hui la plupart des salles ferment comme ce petit cinéma de quartier et se transforment en atelier.

Un changement s'impose, Pietro effectue lui-même les travaux en aménageant la cabine de projection en bureau et en perçant des fenêtres dans la grande salle pour faire entrer assez de lumière pour les ouvrières.

D'habitude, Pietro arrive le premier, il s'intéresse à accueillir lui-même ses ouvrières en répétant à sa fille Giulia 
<<'est ça, être le padrone,...>> (55)

De plus

« Malgré ses accès de colère légendaires, (...) [il] est un père aimant, bien qu'exigeant et autoritaire, [qui] a [bien] élevé ses filles dans le respect de la discipline, ... >> (56)

Ce secteur de production forme la seule source de revenu pour la famille Lanfredi mais également pour les ouvrières qui y travaillent

$<$ voilà près d'un siècle que sa famille

vit de la cascatura, cette coutume

sicilienne ancestrale qui consiste à garder

les cheveux qui tombent ou que l'on coupe

, pour en faire des postiches ou perruques .> $>^{(57)}$

Un jour, Pietro tombe victime d'un accident pendant sa tournée. Pour une raison inconnue, sa vespa quitte la route. Il connaît pourtant ce trajet, il le parcourt plusieurs fois.

À L'hôpital Francesco, il est entre la vie et la mort ce qui emmène les médecins à dire

$<$ Il faut se préparer au pire >> (58)

Mais pour Giulia, elle refuse totalement ce pire ne pouvant l'imaginer

< Un père ça ne meurt pas, un père c'est éternel, c'est un roc, un pilier, surtout le sien .> ${ }^{(59)}$

La mamma demande à Giulia et ses sœurs de l'accompagner $<$ à la procession de Santa Rosalia. >> (60)

Ensemble, toutes rattrapent le cortège . En s'étouffant, Giulia s'éloigne du défilé en se réfugiant dans une petite rue pour se

(La discrimination et ses formes ...) Dr. Abir Chéhata 
raviver de l'eau de la fontaine. Un peu plus loin, elle entend deux gendarmes en uniforme appelle un homme à la peau sombre qui porte un turban noir. Les deux gendarmes

$<<$ haussent le ton, l'un d'eux commence à

le basculer. L'attrapant fermement, ils

finissent par l'entraîner en direction de la gendarmerie .>> (61)

Ce soir, Giulia ne sait pas dormir. L'image de cet homme à la peau sombre lui revient sans cesse. Elle ne peut s'arrêter de se poser plusieurs questions

$\ll$ Une question, plus que toute autre, la tourmente : aurait - elle dî intervenir?

Et qu'aurait-elle pu faire? (..)

Un sentiment étrange s'est emparé d'elle lorsqu' ' il l'a regardée- un sentiment qu'elle ne connaît pas. Est - ce de la curiosité?

De l'empathie?

À moins que ce ne soit autre chose, qu'elle ne sait pas nommer $>>^{(62)}$

Pietro repose dans le fit de l'hôpital pendant huit jours. Ayant l'air tranquille, endormi, sa fille Giulia se rappelle de l'histoire de la Bella Addormentata qu'il lui a lu lorsqu' elle était enfant. Elle aimait beaucoup cette voix résonnante du père

$\ll$ La voix s'est tue. Il n'y a que le silence, à présent, autour du papa. >> (63)

Chaque soir, Giulia va à l'hôpital après la fermeture de l'atelier pour lire au chevet de son père parce que selon les avis des médecins, les malades entendent ce qu'on dit autour d'eux. Alors, Giulia lit des heures à haute voix des romans, de la prose et de la poésie en disant 
$\ll C^{\prime}$ est à moi de lui lire des histoires à présent, (...) Il l'a tant fait pour moi. De là où il est, son papa l'entend, elle le sait. $>$ (64) $^{(64)}$

En outre

$\ll$ À La clameur des discothèques, Giulia préfère le silence feutré de la biblioteca communale. Elle s'y rend chaque jour à l'heure du déjeuner. Insatiable lectrice, elle aime l'ambiance des grandes salles tapissées de livres, que seul le bruissement des pages vient troubler. Il lui semble qu'il y a là quelque chose de religieux un recueillement quasi mystique qui lui plaît.

Lorsqu' elle lit Giulia ne voit pas le temps passer. (...) Elle se dit qu'elle pourrait passer sa vie en cette seule compagnie. $>>^{(65)}$

Ce jour-là, Giulia se rend à la bibliothèque, lieu de refuge et d'isolement pour y emprunter des livres. Alors qu'elle entre dans la salle où se propage le silence. Soudain, elle a perçu cet homme à la peau sombre qui porte un turban noir essayant de chercher un ouvrage mais n'arrive pas à le trouver. Giulia se rapproche de lui le rappelant de l'incident des gendarmes. Il sourit en se demandant :

$<<$ (...) qui est donc cette étrange fille

qui semble s'inquiéter de lui ?>> (66)

Cet homme répond à Giulia en lui disant que les gendarmes

$\ll$ m'ont gardé l'après-midi. Et ils m'ont

laissé repartir.>> (67)

Giulia lui demande s'il cherche un ouvrage en particulier. Il lui explique qu'il veut un livre en italien pas trop compliqué. 
Celle- ci lui conseille d'un roman de Salgari, son préféré de l'enfance. L'étranger le prend et la remercie avant de s'éloigner. Le lendemain, Giulia revient à la bibliothèque espérant de le rencontrer. Elle n'a pas cependant besoin de livres parce qu'elle n'a pas encore terminé ceux qu'elle lit à son père. En entrant dans la salle de lecture, Giulia trouve l'homme à la même place que la vieille.

$\ll$ Il lève les yeux vers elle, comme s'il l'attendait. À cet instant, Giulia a l'impression que son cour va se décrocher.>> (68)

Alors que l'inconnu se prépare à s'en aller, Giulia lui propose d'aller faire quelques pas sur la mer. Celui-ci accepte après avoir marqué un temps. Kamal est différent,il ne parle pas facilement. Il accepte seulement de raconter son histoire à Giulia.

$\ll$ De religion sikh, il a quitté le Cachemire à l'âge de vingt ans, fuyant les violences faites aux siens là-bas. Depuis les événements de 1984, lorsque l'armée indienne a réprimé dans le sang les revendications des indépendantistes, massacrant les fidèles dans le Temple d'Or, leur sort est menacé. Kamal est arrivé en Sicile par une nuit glacée, sans ses parents- >> (69)

En plus, Kamal relate

$<$ comment le caporale recrute et achemine les clandestins vers leur lieu de travail. Pour couvrir les frais de déplacement, la bouteille d'eau et le 
maigre panino qu'il leur donne, il prend un pourcentage de leur salaire, parfois jusqu'à la moitié.>> (70)

Kamal et Giulia se voient tous les jours, Giulia s'influence par cet homme qui diffère des siciliens qu'elle connaît. Un midi, elle l'emmène vers une plage où les touristes n'y vont pas. Elle veut lui indiquer une grotte que personne ne connaît.

$<$ Ils prennent l'habitude de se retrouver là, dans la grotte, près de la mer. (...) ils se voient à l'heure du déjeuner.Ils font l'amour à midi, (...) ces heures-là leur appartiennent ...>> (71)

Giulia sait que Kamal n'a pas la même peau ni le même dieu que les Lanfredi. Alors, elle décide de l'aimer secrètement. Leurs amours sont cachés. Ce sont des amours sans papiers.

Un jour, la mamma livre à Giulia une mission, celle de chercher un papier dans le bureau de son père à l'atelier. L'hôpital revendique un document qu'elle ne m'arrive pas à trouver. Giulia n'ose pas refuser. Elle n'a pourtant pas envie d'entrer dans ce bureau.

Elle ne veut pas toucher aux papiers de son père tenant à ce qu'il retrouve la pièce telle qu'il l'a laissée. Giulia pousse la porte du bureau mettent un temps à y entrer. Doucement, elle ouvre un premier tiroir puis un deuxième. Le troisième est fermé à clé. Après de courtes recherches, elle finit par trouver la clé

D'une main frémissante, elle donne un tour de clé. Le tiroir s'ouvre finalement : il contient un tas de papiers, Giulia s'en s'empare

(La discrimination et ses formes ...) Dr. Abir Chéhata 
$\ll$ La vérité la frappe comme une gifle. Elle tient en un mot :faillite. L'atelier croule sous les dettes. La maison Lanfredi est ruinée.>>(72)

En plus

$\ll$ D'après les comptes, il reste un mois de travail, tout au plus. Sans cheveux, les ouvrières vont se retrouver au chômage technique. l'atelier ne pourra plus les payer. Il faudra déposer le bilan, et fermer. >> (73)

Cette idée accable Giulia elle pense à sa sœur Adela qui est encore au lycée, à sa sœur aînée Francesca, femme au foyer que son mari gaspille son salaire au jeu et à son père

$<$ Que vont - ils devenir ? La maison familiale est hypothéquée, tous leurs biens vont être saisis. >>(74)

Giulia choisit de quitter le lycée pour aider son père à l'atelier. Élève douée selon ses professeurs, elle aurait complété ses études et entrer à l'université

$<$ Mais il était pour elle impensable de changer de voie. (...) Étrangement, les sæurs de Giulia n'ont pas manifesté d'intérêt pour le métier, et elle est la seule des filles Lanfredi à s'y consacrer.>> (75)

De plus

$<(.$. ) tout est calme dans l'atelier. (...) il n'y a que le silence, et l'écho des pas de Giulia. Elle marche jusqu'au vestiaire réservé aux ouvrières, et dépose ses affaires dans le casier à son prénom. Elle attrape sa blouse, se glisse comme chaque jour dans cette seconde peau. Elle 
rassemble ses cheveux, les roule en un chignon serré(...) Ainsi vêtue et coiffée, elle n'est plus la fille du patron :elle est une ouvrière comme une autre, une employée de la maison Lanfredi. (...) Elle a toujours refusé d'être privilégiée..>> (76)

Giulia n'ose pas parler aux ouvrières ni leur mentir. Aussi, elle ne peut pas travailler à côté d'elles comme si rien ne se passait. À midi Giulia rejoint Kamal dans la grotte où ils font l'amour. Elle ne dit rien de ses tourments.

Le soir, elle regagne la maison familiale sous prétexte d'une migraine, elle s'enferme dans sa chambre Au malheur de son père et à la mésaventure touchant la vie financière causant la ruine de l'atelier Giulia voit cette nuit tourner au cauchemar.

$\ll(.$.$) l'atelier de son père démembré ,la$ maison vidée, vendue, sa mère hagarde, les ouvrières à la rue,les mèches de la cascatura dispersées, jetées dans la mer, (...) Giulia se tourne et se retourne, (...) mais les images reviennent inlassablement, comme un rêve obsédant dont elle ne parvient pas à se libérer...>> (77)

Nous sentons, nous lecteurs, que Giulia se trouve dans une impasse, elle ne sait quoi faire, de même elle ne sait comment se débrouiller c'est à elle seule que compte le sauvetage de l' atelier

$<$ (..) le rapport émotionnel envers le héros relève de la construction esthétique de l'œuvre et ce n'est que dans les formes primitives qu'il coïncide obligatoirement avec le code traditionnel de la morale et 
de la vie sociale.Autrement dit, le textuel prime l'idéologique: la dimension affective du personnage est d'abord liée aux modalités de sa $<<$ mise en texte $>>$. (78)

Giulia annonce à sa mère et ses sœurs la ruine de l'atelier. Francesca se met à pleurer, Adela ne prononce aucun mot, la mamma s'effondre en se demandant

<Quel crime ont-ils commis, quel péché pour mériter ce châtiment ?! Que vont devenir ses enfants ?>> (79)

Puis, une idée lui vient à la tête évoquant Gino Battagliola, cet amoureux de Giulia depuis longtemps, il rêve de l'épouser. Sa famille est riche possédant plusieurs salons de coiffure. Ses parents prouvent souvent une fidèle amitié aux Lanfredi. Ils accepteraient donc de racheter l'hypothèque de la maison familiale.

Giulia s'enferme dans sa chambre ne pouvant se soumettre à ce choix.

$$
\begin{aligned}
& \text { < Quelle autre issue, pourtant ? Va-t- } \\
& \text { elle accepter que sa mère et ses sœurs se } \\
& \text { retrouvent à la rue? La vie est cruelle, se } \\
& \text { dit- elle, de faire ainsi peser sur ses seules } \\
& \text { épaules le poids de sa famille entière.>> } \\
& \text { (80) }
\end{aligned}
$$

Vraiment, Giulia est discriminée par la ruine de l'atelier, c'est elle, seule, la responsable de trouver une solution à ce problème financier et de sauver sa famille.

Après avoir réfléchi plusieurs fois, elle arrive à la seule solution proposée parsa mère celle de se marier avec Gino.

$\ll$ Si l'atelier est condamné, sa famille, au moins, sera sauvée. C'est ce que dit sa 
mère, et ce qu'aurait voulu le papa. Cet argument achève de convaincre Giulia $>{ }^{(81)}$

Le soir même, Giulia écrit une lettre à Kamal dans laquelle elle lui explique les pires circonstances de l'atelier, la menace pesant sur sa famille et sa décision de se marier mais cette lettre elle ne sait ni où l'envoyer ni l'endroit où Kamal vit. Giulia finit par la laisser dans la grotte sous un coquillage .Giulia doit annoncer la fermeture de l'atelier aux ouvrières, elle est obligée de les renvoyer

$\ll$ Il n'y aura rien pour apaiser leur peine, juste des larmes amères à partager. (..)Que va devenir la Nonna? Qui voudra la réengager? Alessia, Gina, Alda ont plus de cinquante ans, un âge critique pour le marché de l'emploi. Que va faire Agnese, seule avec ses enfants depuis que son mari l'a quittée ? Et Federica, qui n'a plus ses parents pour l'aider? .... >> ${ }^{(82)}$

Giulia veut repousser l'idée d'annoncer la mauvaise nouvelle de même, elle doit leur dire, cette pensée la rend frustrée et la demeure éveillée.

En pleine nuit, un caillou jeté à sa fenêtre par Kamal qui incite Giulia de descendre,pour lui parler dans la rue .

$<$ Il a lu sa lettre, dit-il: la fermeture de l'atelier n'est pas une fatalité .Il y a une solution qui pourrait les sauver. $>>^{(83)}$

\section{Discrimination de L'état de santé}

Dans l'histoire de Sarah, nous retrouvons une hiérarchie qui est établie dans ce cabinet d'avocats où la compétition est 
tellement féroce. Sarah, elle-même est insensée dans l'entrainement de cette hiérarchie.

$\ll$ S'il y a une majorité de femmes parmi les collaboratrices, Sarah est la première à avoir été promue associée, dans ce cabinet réputé machiste. >> (84)

En outre

$<$ Certaines \{de ses amies\} ont même abandonné, changé de carrière, malgré les études longues et exigeantes qu'elles ont menées. Mais pas-elle. Pas Sarah Cohen. Le plafond elle l'a pulvérisé, fait exploser à grands coups d'heures supplémentaires, des Week-ends passés au bureau, de nuits à préparer ses plaidoiries.>> (85)

Rappelant son entretien d'embauche, elle s'est retrouvée devant huit hommes dont Johnson

$\ll$ en personne, l'associé fondateur, le Managing Partner, Dieu lui - même, sorti de son bureau pour l' occasion, et descendu en salle de réunion. >> (86)

Au cabinet la nouvelle est annoncée qu'elle a été la prochaine Managing Partner.

Sarah avait tout pour être choisie

$\ll$ un parcours exemplaire, une volonté sans faille, une capacité de travail défiant toute concurrence - une forme de boulimie qui toujours la poussait à rester en mouvement.(...) 
Ce jour-là, elle l'attendait sans vraiment l'espérer. Bien sûr, sa carrière avait exigé des sacrifices. Elle lui avait coûté son lot de nuits blanches, et ses deux mariages. $>>(87)$

Dans un entretien, Colombani nous indique

$\ll$ A travers le portrait de Sarah, j'ai voulu illustrer le profond dilemme de nombreuses femmes de notre société occidentale, et l'écartèlement permanent dans lequel elles vivent. Elles doivent être des mères parfaites, des épouses modèles assumer toutes les tâches domestiques et réussir brillamment leur carrière. C'est une position intenable .La société leur demande trop, et ne leur fait pas de cadeaux. On pardonne à un homme de délaisser son foyer pour se consacrer à son métier; pas à une femme. >> (88)

Sarah a favorisé sa vie professionelle au détriment de sa vie conjugale et familiale. Près de ses collaborateurs et ses associés, Sarah n'a laissé rien apparaître ,elle avait une loi stricte de ne plus parler de ses enfants :lorsqu' elle était obligée de quitter le cabinet pour une consultation chez le pédiatre ou un appel à l'école, elle chois irait dire qu' elle avait plus d'un rendez-vous extérieur

< Il valait mieux mentir, inventer, broder, tout, plutôt qu'avouer qu'on avait des enfants, en d'autres termes: des chaînes, des liens, des contraintes. Ils étaient autant de freins à votre 
disponibilité, à l'évolution de votre
carrière. >> (89)

Dans l'ancien cabinet où Sarah travaillait une femme venant d'être promue associée,

$\ll \grave{A}$ l'annonce de sa grossesse[elle]

s'était vue destituée, renvoyée au statut de collaboratifs. (..)Sarah en avait tiré une leçon pour elle-même. Lors de ses deux grossesses, elle n'avait rien dit à sa hiérarchie.>> (90)

Le poste ainsi que le grade prennent une place primordiale dans la vie de Sarah. Sa vie professionnelle est une quête obstinée d'un posteà atteindre.

$<$ Elle traçait son chemin, (..)laissant

[les hommes] qui détestaient les femmes sur le bas-côté. Chez Johnson\& Lockwood, elle avait gravi les échelons à la vitesse d'un cheval lancé au galop, se forgeant une solide réputation en cours de justice.>> (91)

À huit heures vingt exacte, elle gare sa voiture dans le parking, face à l'affiche portant son nom $:<<$ Sarah Cohen, Johnson \& Lockwood >>

Cette affiche qu'elle médite chaque matin avec honneur montre plus que

$<<$ l'emplacement de sa voiture; elle est un titre, un grade, sa place dans le monde. Un accomplissement , le travail $d^{\prime}$ une vie. Sa réussite, son territoire. >> (92)

Notons encore que

$$
\begin{aligned}
& <<\text { (...) le personnage romanesque a } \\
& \text { toujours une fonction référentielle de }
\end{aligned}
$$


première importance en raison du rôle qu'il joue. (...) il représente des existences en devenir dans un récit qu'il contribue à fabriquer, à infléchir, bref à structurer autour de situations et d'actions: tout comme il ne saurait exister de roman sans actions, il ne peut $y$ avoir d'action sans personnage ... >> (93)

Ce qui nous oblige ici à rappeler ce que Max Weber le sociologue allemand appelle 《< le prestige de la profession >>

$\ll$ Max weber complexifie son analyse de la stratification sociale en y ajoutant une analyse des Ständische Lage (...), expression traduite ordinairement par sa situation statutaire ou groupes de statut (...) $<<$ Nous appelons $<<$ conditions $>>$ (...) un privilège positif ou négatif de considération sociale revendiqué de façon efficace fondé sur le mode de vie, le type d'instruction formelle (..) le prestige de la naissance ou le prestige de la profession>> (94)

Un jour, Sarah tombe au milieu d'une plaidoirie. Immédiatement, elle ne savait guère où elle était. Elle a essayé de reprendre sa démonstration malgré la pâleur de son visage et le frémissement de ses mains qui seuls dupaient son embarras.

$<<$ (...) Sa chute s'est faite en silence.

Elle n'a pas protesté, n'a pas appelé à

l'aide. Elle s'est écroulée sans un bruit, comme un château de cartes, presque avec grâce. >> (95) 
Après avoir l'emmenée à l'hôpital, l' interne qui l'examine lui pose plusieurs questions Sarah répond de façon brève. Elle répète qu'elle va bien mais celui-ci continue son examen Sarah ne dit plus la vérité

$<$ Elle ne dit pas non plus cette douleur dans la poitrine du côté gauche, qu'elle ressent depuis quelque temps. Sans doute rien ... Elle n'a pas envie d'en parler, pas là, pas maintenant, (...) Ce n'est pas le moment.>> (96)

L'interne paraît inquiet, il lui propose de se reposer, de prendre un congé. Mais pour Sarah, elle refuse cette suggestion en se demandant comment faire ?

$\ll$ En vendant ses enfants sur eBay?

(...) En annonçant à ses clients qu'elle

fait grève au cabinet ? Elle gère des dossiers aux enjeux cruciaux, qu'elle ne peut déléguer. S'arrêter n'est pas une option. > (97)

En entendant ces questions, l'interne ne trouve que cette phrase à lui échapper

< personne n'est irremplaçable. >> (98)

Il n'pas la moindre idée de ce que veut dire

$\ll \quad$ être associée chez Johnson

\&Lockwood. Aucune idée de ce que veut

dire: être dans la peau de Sarah Cohen.>> (99)

L'interne essaye de la retenir pour d'autres examens mais elle fuit rapidement. Ainsi, Sarah revient à son monde, et ses plaidoiries, à ses dossiers, à ses comptes rendus et à ses trois enfants. 
$\ll$ Elle retourne au front comme un bon petit soldat, remet ce masque qu'elle a toujours porté et qui lui va si bien, celui de la femme souriante à qui tout réussit. (...) En arrivant au cabinet, elle rassurera Inès et ses collaborateurs : ce n'était rien. Et tout repartira comme avant. >> (100)

Dans les semaines suivies, durant une visite de contrôle chez la gynécologue, elle recommande à Sarah une liste d'examens aux noms brutes

< mammographie, IRM, scanner, biopsie > (101) [ces examens] sont presque un diagnostic. Une condamnation. >>(102)

La bombe s'est explosée dans le cabinet du médecin, cet homme lui parle

$<$ de la tumeur sur ses radios, la taille d'une mandarine (...) c'est un mot que personne ne prononce, un mot qu'il faut deviner, derrière les périphrases, le jargon médical dans lequel on la noie.>> (103)

Sarah écoute le médecin mais ses mots ne paraissent pas la toucher. Indifférente, pour elle - même, il n'en est rien.

A la fin de cette visite, Sarah ne demande pas ses chances, elle repousse l'idée de réduire son futur à une statistique, elle ne laisse pas les chiffres s'insinuer en elle, ils seraient capables de se multiplier, comme la tumeur elle - même, de détruire son moral, sa confiance et sa guérison

$\ll$ Elle est une guerrière. elle va se

battre. (...) Il s'agit d'une procédure au

long cours, ce sera une guerre des nerfs,

(La discrimination et ses formes ...) Dr. Abir Chéhata 
une succession de moments d'espoir, de doute, et d'autres(.....)Il faudra tenir, coûte que coûte. Ce genre de combat se gagne à l'endurance, Sarah le sait.>> (104)

Comme elle étudie un dossier, elle organise son plan d'attaque de la maladie. Elle n'avoue rien à personne au cabinet.

La nouvelle serait l'effet de l'explosion d'une bombe chez les collaborateurs, les associés et encore chez les clients $<<$ Sarah est l'une des fondations $d u$ cabinet, l'un de ses piliers, elle doit rester solide pour ne pas faire pencher l'édifice tout entier. >> (105)

Lorsqu'elle retourne au cabinet, Sarah reprend ses travaux secrètement, elle guette la réaction de ses collègues et leurs regards, elle vérifie avec apaisement

$<$ que personne n'a rien remarqué. Non, elle n'a pas le mot <<cancer >> gravé sur le front, personne ne voit qu'elle est malade. À l'intérieur elle est en miettes, mais cela, personne ne le sait.>>(106)

Au début, la stratégie de Sarah est bien organisée. Elle a pris deux semaines de congé pour l'opération il en faut trois - le médecin a pourtant accentué, une semaine d'hospitalisation en plus deux de repos, qu'elle a diminuées à une. Sarah ne peut pas prendre un long congé sans provoquer les doutes au cabinet.

Sarah n'a rien dit au bureau, ni à son père ni à son frère. $\mathrm{Sa}$ mère est morte de la même maladie c'est pourquoi elle ne désire pas leur dicter de nouveau ce circuit du combattant. Alors, elle décide de se battre seule silencieusement. Quant à

(La discrimination et ses formes ...) Dr. Abir Chéhata 
ses enfants, elle leur a d'abord expliqué qu'elle était obligée de subir

$$
\begin{aligned}
& \ll<\text { une intervention }>, \quad<\text { Sans } \\
& \text { gravité }>>(107)
\end{aligned}
$$

A - t-elle amplifié, pour ne pas les rendre inquiets. Puis, Sarah a déterminé qu'ils ne pourraient pas lui rendre visite à l'hôpital sous prétexte que les enfants n'y étaient pas autorisés

$\ll$ Elle veut les préserver de cet endroit, cet enfer blanc aux odeurs âcres (...) ce sont les odeurs qui l'indisposent à l'hôpital, ce mélange de désinfectant et de Javel qui lui noue l'estomac. Elle ne veut pas que ses petits la voient ainsi, vulnérable, affaiblie. >> (108)

$\mathrm{Au}$ cabinet, Inès, seule, a trouvé Sarah épuisée lorsqu' elle est retournée de congé. Heureusement, c'est l'hiver, les corps sont recouverts de pulls et de manteaux. Sarah s'intéresse à ne plus porter de décolleté, à se maquiller encore plus qu'avant elle a mis par excellence un inventif système de code

$\ll$ il y a un sigle pour les séances à l'hôpital (RDVH), un autre pour les examens, prélèvements et radios, qu'elle place toujours entre midi et deux (Déjeuner R), et ainsi de suite. >> (109)

Ses collègues vont croire qu'elle a un amant, qu'elle va le rencontrer à l'heure du déjeuner. Quant à l'équipe des juniors, les discussions vont d'une rapide vitesse

$\ll$ Est - ce un collègue ? Un associé ?

Inès penche pour un homme marié, (...)

Pourquoi tant de précautions, sinon? 
Imperturbable, Sarah continue ses allées et venues. Son plan semble fonctionner. $>$ (110)

La maladie de la mère d'Inès a été dite à Sarah mais cette information s'était perdue dans sa tête débordée. De même, elle a oublié la permission donnée à Inès de prendre sa journée

$<$ depuis quelques temps, certaines choses lui échappent, son état de fatigue avancé en est sûrement la cause. >>(111)

Les deux sa rencontrent dans la salle d'attente du service d'oncologie de l'hôpital universitaire. Une même expression de stupéfaction se verra sur leur visage. Sarah reste muette

$\ll$ Pour se donner une contenance, Inès

lui présentera à sa mère >> (112)

Sarah ne laissera rien apparaître de son bouleversement - que fait-elle sa directrice dans cet endroit en tenant des radios sous le bras.

Dans une tentative un peu inutile pour sauver le situation, Sarah réclame qu'elle s'est trompée de salle, elle est venue visiter une amie. Mais elle est sûre qu'Inès va vite reconstruire le puzzle : son absence de quinze jours le mois dernier, les rendez - vous, son teint, sa maigreur, autant de signes qui sont des preuves de persuasion.

Sarah se sent stupide de frémir devant une collaboratrice junior, comme si elle était coupable

$<$ Elle a un cancer, ce n'est pas un crime. Et puis elle n'a pas à se justifier auprès d'Inès, elle ne lui doit rien, ni à elle, ni à personne. >> (113) 
Sarah décide d'assigner Inès à son bureau et de lui proposer de l'aider dans l'affaire Bilgouvar, le fameux dossier du moment pour le client le plus primordial du cabinet.

$\ll$ Une habile façon d'acheter son silence, se dit-elle, de s'assurer sa loyauté. Inès est ambitieuse, elle comprendra qu'elle n'a pas intérêt à parler, à s'attirer les foudres de son associée.>>(114)

Sarah ressent un changement au cabinet. D'abord, un regard, puis une façon de savoir ses nouvelles ou au contraire de ne rien questionner, ensuite une manière de la contempler $\ll$ Aucun n'est naturel. $>^{(115)}$

Il suffit seulement d'un mot prononcé à voix tout bas par Inès avec un regard affligé 《je suis désolée»(116)

Sarah ne prend pas du temps à comprendre qu'elle est $\ll$ la victime, la blessée l'endeuillée. >>(117)

Inès a brisé le pacte sans avertir, elle a exhibé son secret. Inès que Sarah a elle-même préféré, trié. Inès avec qui elle partage ses documents. Oui, c'est Inès qui l'a lardée, de la façon la plus indigne, la plus ignoble qui soit

\begin{abstract}
$\ll$ La nouvelle s'est répandue dans le cabinet comme une étincelle sur de la poudre, elle a longé les couloirs, envahi les bureaux, s'est diffusée dans les salles de réunion, à la cafétéria, jusqu' à parvenir au dernier étage, tout en haut de la hiérarchie, auprès de Johnson. >> (118)
\end{abstract}


Inès a confié le secret à la personne chatouilleuse de

la dévoiler Gary Curst le plus envieux, le plus ambitieux, le plus arriviste, le plus $\ll$ misogyne des associés >> (119) qui consacre à Sarah une profonde rancune, effrayante depuis sa venue. Encore, Inès lui a dit que Sarah perpètre des fautes dans le dossier Bilgouvar

$<<$ Des faux pas, des erreurs, elle n'en a pas commis, Inès le sait. Alors pourquoi? Pourquoi donc la trahir?

Sarah le comprend trop tard, et cette pensée la glace: Inès veut sa place. Son statut d'associée.>> (120)

Les chances de promotion sont faibles au cabinet, on ne livre pas aisément les juniors escalader en poste. Un associé abattu n'est qu'une chance à ne pas manquer. Curst y trouve le même profit : il a souvent envié la liaison liant Sarah à Johnson

$<$ Elle est sans doute la prochaine Managing Partner qu'il va nommer. À moins que quelque chose ne vienne freiner son ascension .... Il s'y verrait bien, Gary Curst, dans ce fauteuil, tout en haut de la hiérarchie. >> (121)

En outre

$\ll$ une maladie vicieuse, pernicieuse, qui vous attaque, vous affaiblit, une maladie susceptible de partir et de revenir, c'est l'arme idéale pour abattre un ennemi. Curst n'aura même pas de sang sur les mains; le crime est parfait. Comme aux 
échecs, un pion tombe, et tous avancent d'une case. Ce pion-là, c'est Sarah.>>(122)

Dans son bureau, là - haut, Johnson l'a mandée. Il aimerait savoir la nouvelle de la bouche de Sarah. Ils ont souvent eu une liaison de confiance, pourquoi n'a- t- elle rien affirmé ?

Sarah distingue pour la première fois

$<$ Cet air condescendant, faussement paternaliste qu'il prend avec elle, et qu'à bien y réfléchir il a toujours pris, elle le vomit.>>(123)

Avec son air de fausse angoisse, Sarah sait bien ce qui l'obsède. Ce n'est ni de savoir comment va-t-elle ? ni comment sera -t-elle encore dans un an ? tout ce qui l'importe, c'est de savoir si elle sera capable de gérer ses dossiers comme avant. En un seul mot : d'être compétitive.

Certainement, Sarah ne dit aucun mot. Elle essaye de rassurer Johnson : non, elle ne décide pas prendre de congé de longue durée. Elle ne va non plus s'absenter. Elle sera au cabinet, malade peut -être mais elle assumera ses responsabilités et poursuivre ses dossiers.

En écoutant ses paroles, Sarah a l'impression d'être au tribunal devant un juge. Elle recherche des raisonnements pour renforcer sa défense.

$\ll$ Est - elle coupable de quelque chose

$?$ !

A-t-elle commis une faute?

De quoi doit-elle se justifier?>> (124)

En retournant à son bureau, elle essaye de se convaincre que rien ne va modifier mais à la réalité, elle sait que Johnson a débuté à démarrer son procès. 
$<$ L'ennemi, songe -t-elle alors, n'est
peut-être pas celui qu'elle croyait. $>>^{(125)}$

L'intrigue manœuvrée contre Sarah, commence de manière mensongère. D'abord, par une assemblée à laquelle on néglige de la convier sous prétexte de ne pas la déranger. Puis, un dossier dont on fuit de lui parler. Sarah veut continuer à travailler, être estimée comme avant. Elle rejette qu'on l'économise. Elle sent qu'on l'introduit moins dans la vie du cabinet, dans les jugements à prendre, la gestion des dossiers. Il y a des choses qu'on omet de lui communiquer, des questions qu' on va demander à d'autres.

Depuis l'annonce de sa maladie, Sarah voit Curst causer avec Johnson, pouffer à ses plaisanteries, l'amener à ses déjeuners . Quant à Inès, elle prend de plus en plus d'indépendance dans les dossiers qu'elle aborde sans demander à Sarah. Elle bénéfice de ses absences pour intervenir dans les réunions en prenant les décisions à sa place, elle s'est beaucoup confrontée Curst, elle s'est mise même à fumer dans le seul objectif de partager avec lui les pauses cigarettes.

Malgré l'opinion de l'oncologue, Sarah repousse de prendre congé. S'absenter signifie laisser sa place, livrer son terrain or elle doit rester debout

$<$ Elle ne laissera pas le cancer lui prendre ce qu'elle a mis des années à construire. Elle va se battre, bec et ongles, pour garder son empire.>> (126)

L'oncologue l'a pourtant prévenue : le traitement sera accablant et de plus ses effets secondaires. Il en a posé une liste totale, dans un cadre qu'il lui a donné, déterminant à quel instant elle sentira des nausées, quelles suites pour ses 
cheveux, ses ongles, ses sourcils, sa peau, ses mains et ses pieds. Sarah est retournée

$<<$ avec une dizaine d'ordonnances, une

pour chaque effet à contrecarrer. >>(127)

Ce que l'oncologue n'a pas déclaré, ce que personne n'a suggéré l'effet auquel elle n'était pas s'apprêtée et qu'aucune ordonnance ne pourra soigner

$<$ c'est l'exclusion qui va de pair avec la maladie, cette lente et douloureuse mise à

l'écart dont elle est devenue l'objet.>>(128)

$\mathrm{Au}$ début, Sarah ne veut pas définir ce qui se déroule au cabinet. Elle préfère méconnaître les $\langle<$ oublis $>>$ de ses collègues ainsi que cette indifférence récente de Johnson.

Cette violence porte une définition qu'elle sent du mal à articuler discrimination Un mot qu'elle a centaines de fois entendu pendant ses procès et qui ne l'a guère effectivement concernée- du moins le pensait - elle.

Se lutter, oui, mais comment ? convoquer Johnson \& Luckwood pour discrimination ? cela exige de démissionner. Si elle part, elle ne m'obtiendra ni aide ni protection sociale. Retrouver du travail autre part ? Qui les embaucherait? Elle et sa maladie ? établir son propre cabinet ? une vision attrayante mais qui implique des engagements, des financements. Les banques prêtent seulement aux gens en bonne santé. Sarah le sait bien. En plus, quels clients la poursuivaient ? Elle ne pourrait rien certifier, pas également qu'elle sera présente dans un an pour défendre leurs dossiers. Sarah se rappelle de ce procès horrible il y a quelques années celui de cette femme défendue par l'un de ses collègues, qui travaillait comme secrétaire dans un cabinet médical.

(La discrimination et ses formes ...) Dr. Abir Chéhata 
S'accusant de douleur à la tête, elle s'était fiée au médecin qui l'employait; il l'avait donc examiné. Après lui avoir passé un examen, il l'avait appelée le soir même, pour lui faire connaître son licenciement : elle avait un cancer .La procédure s'était prolongée trois ans, la femme avait fini par gagner mais elle était morte quelques temps après.

Un matin du mois de janvier, Johnson l'appelle dans son bureau Il prend de ses informations, l'air faussement ému. Il rappelle alors ce cousin éloigné, traité par un cancer il y a vingt ans, qui est en bonne forme aujourd' hui .

Sarah s'enfiche de toutes ces guérisons qu'on lui lance au visage. Elle voudrait lui répliquer que sa mère en est morte, qu'elle -même est malade

$\ll$ comme un chien, que sa fausse compassion, il peut se la garder. Il ne sait pas ce que c'est, d'avoir des aphtes dans la bouche au point de ne pas pouvoir manger, de sentir vos pieds si brûlants \{ que\} vous ne pouvez plus marcher, (...) que dans quelques semaines vous n'aurez plus de cheveux, que votre corps est tellement maigre (...) que vous avez peur de tout, peur de souffrir, peur de mourir, (..) $Q u^{\prime}$ il aille donc se faire foutre, avec sa bonne conscience. Et son cousin aussi >> (129)

Arrivant au dossier Bilgouvar, Johnson veut lui attribuer un associé mais il est son client il y a des années, elle n'a besoin de personne, pour gérer son dossier. Johnson lamente en évoquant l'unique assemblée où elle est arrivée en retard. Puis, 
$<<$ (...) Il prend alors cette voix mielleuse, (...) pour lui dire qu'il comprend - qu'elle- ait -des- obligations - médicales, que tout -le-monde -icisouhaite- qu'elle -se -rétablisse -au mieux -et -au -plus - vite, (...) ces phrases toutes faites qui ne veulent rien dire,qui sonnent creux, il pense que Sarah-a-besoin-d'être-soutenue, c'est la-vocation - et-l'essence - même - dece-cabinet, du-travail - en -équipe. Pour-l'épauler - dans- ce-moment difficile - il - va-lui-adjoindre- $l^{\prime}$ aide - de ... Gary Curst. >> (130)

Sarah préférait être frappée, insultée, elle sait bien qu'il est inutile d'argumenter, rien ne modifiera l'ordre des choses.

$\ll$ Son sort est scellé, Johnson en a décidé. Malade, elle ne lui est plus d'aucune utilité. Elle est une valeur sur laquelle il ne veut plus compter. >> (131)

Nous jugeons que Sarah incarne l'idée de $\langle<$ réification $>>$ ou $<<$ chosification $>>$ pour affirmer - qu' elle est minimisée à l'état de chose.

$<[.$.$] dans sa signification et son usage$ standard, le terme est invariablement accompagné par de fortes connotations négatives. Dès lors, il désigne le devenirchose de ce qui, en droit, n'est pas une chose. Cette pseudo -chose peut être, selon le cas, un concept, une personne une relation, un processus, le monde social, une marchandise,etc.- (..) La réification de ces pseudo - choses 
consiste à leur attribuer illégitimement, et, selon le cas, une facticité, une fixité, une objectivité, une externalité, une impersonnalité, une naturalité; bref, une choséité ontologique qui est jugée inappropriée. >> (132)

Aussi, la $<<$ réification $>>$ est un terme marxiste faisant l'objet des recherches

détaillées chez Luckás :

$<[.$.$] il importe de souligner que la$ notion de réification a une histoire dans la pensée de Luckás. Dès son premier ouvrage , L'Histoire du développement $d u$ drame moderne (1908), il dénonce par exemple la $<<$ chosification de la vie $>>$ , la $<$ tendance à la dépersonnalisation et à la réduction du qualificatif au quantitative $>>(\ldots)<<$ ce désir de tout réduire à des chiffres et des formules >>,[ dominant] l'ensemble de la vie >> (133)

Luckás raisonne que

$<$ dans la sphère du processus de production et de reproduction matérielle, l'expression la plus achevée de la réification est la transformation de l'homme en marchandise et en appendice de la machine >>. (134)

Nous constatons que le travail de Sarah qui l'a réduite à une chose. Son cerveau est comparé au

$<<$ processeur d'un ordinateur. $>>(135)$

Alors quand 
$\ll L^{\prime}$ alarme sonne et le compte à rebours commence. Sarah est en lutte contre le temps, de l'instant où elle se lève à celui où elle se couche. À la seconde où elle ouvre ses yeux, son cerveau s'allume comme le processus $d^{\prime}$ un ordinateur.>> 136)

En n'étant plus énergique, plus productive, elle s'est vue discriminer de ses collègues du cabinet.Elle est telle qu'une machine de production que l'on en profite et que l'on jette à la fin après s'être tombée malade d'un cancer.

Cette $<<$ réification $>>$ d'après Marx, influence les rapports sociaux entre les individus. En minimisant l'être humain à l'état de chose. Ce sont les rapports sociaux ici qui se sont disloqués

$<<(\ldots)<$ Dans la valeur d'échange, la relation sociale des personnes est transformée en un comportement social des choses; le pouvoir de la personne s'est transformé en pouvoir de choses>> (...) [Aussi], il précise que $\ll$ ces rapports objectifs de dépendance, par opposition aux rapports personnels, apparaissent encore sous un autre aspect [...]: désormais les individus sont dominés par des abstractions,alors qu'antérieurement ils dépendaient les uns des autres $>>{ }^{137)}$

Curst va s'emparer du dossier Bilgouvar, le plus gros client de Sarah. Ensemble, les trois Inès, Curt et Johnson vont la morceler, la démembrer, alors qu'elle est déjà à terre. Elle voudrait crier à haute voix mais il n'y aura personne ni l'entendre ni lui venir en aide

(La discrimination et ses formes ...) Dr. Abir Chéhata 
$<$ C'est une violence chic, une violence parfumée, une violence en costume trois pièces .>138)

Sarah savait qu'elle en recevait des coups mais elle n'attendait jamais à celui - là.

Après quelques jours, quand la nouvelle s'annonce, elle ne l'étonne même pas : Curst est mentionné Managing Partner.

Cette mention $<$ sonne le glas du carrier de Sarah.>> (139)

Ce jour - là, elle retourne chez elle en milieu d'après-midi , une heure qu' elle ne connaît plus. Elle s'assoit sur son lit en se mettant à pleurer car elle rappelle

$<<$ cette femme qu'elle a été, qu'elle était hier encore, une femme forte et volontaire qui avait sa place dans le monde, et se dit qu'aujourd'hui, le monde l'a abandonnée (...) D'abord, il y a eu le déni, l'incrédulité. Puis la colère, (..) L'abattement lui a succédé, incommensurable, comme une étendue désertique n'offrant pas d'échappée. >> (140)

Sarah se sent leurrée, comme une femme repoussée qu'on rejette parce qu'elle n'a pas donné ce qu'on espérait d'elle, parce qu'on la juge incompétente, déficiente et stérile. Johnson ainsi que ses collaborateurs ont décidé de l'enterrer. Ils ont éjecté son corps faible, fragile dans un fossé en l'ensevelissant doucement. À vrai dire, Sarah est morte professionnellement, elle le sait très bien. Son planification n'a pas fonctionné. Son mur s'est écroulé, excite par l'aspiration d' Inès, multipliée de celle de Curst avec la protection de Johnson. Ils lui ont arraché l'unique chose qui la retenant debout, l'unique qui cédait à la force de se 
réveiller chaque matin : son moi social, sa carrière, l'impression d'avoir sa place dans le monde.

$<$ Sarah est devenue son cancer. Elle est sa tumeur personnifiée. (...) Pour eux elle $n^{\prime}$ est plus une avocate malade, elle est une malade avocat. (...) Le cancer fait peur. Il isole, il éloigne. Il pue la mort. (...)

Intouchable, voilà ce que Sarah est devenue. Reléguée au ban de la société.>> (141)

Dans ce cas non, elle ne retournera plus au cabinet. Ils ne la verront jamais chuter elle ne se présentera jamais en pâtures à ces animaux sauvages. Il lui reste de plus : sa dignité . Elle a passé ses matinées à regarder le film des dernières semaines en se demandant ce qu'elle aurait pu exécuter pour modifier le déroulement

$\ll$ Rien, sans doute. La partie s'est jouée sans elle. Game Over. Terminé. (...) Elle pensait gérer la maladie à la manière d'un dossier, avec méthode application et volonté :Cela n'a pas suffi> ${ }^{(142)}$

Ce qui blesse Sarah Cohen, ce qui la déchire ce n'est pas la maladie qui a pénétré de son corps non, ce qui la tue vraiment c'est le renoncement de ceux qu'elle estimait comme ses pairs dans ce cabinet dont elle a participé à faire la réputation.

Dans un livre sur les animaux, destiné aux enfants, Sarah a lu un jour cette phrase: 
$<$ Les carnivores sont utiles à la nature, car ils dévorent les faibles et les malades.>>(143)

Sarah a rassuré sa fille qui s'est mise à gémir, à se lamenter par lui dire que

$\ll(\ldots)$ les humains n'obéissaient pas à cette loi. (...) Alors on peut lui en prescrire, des pilules, autant qu'on veut, elles ne changeront pas grand-chose, ou si peu. Il y aura toujours des Johnson et des Curst pour lui remettre la tête sous l'eau .

Bande de salauds. > (144)

Cette guerre-là, Sarah n'est pas certaine de la gagner. Ce corps qu'elle a pendant des années assujetti, méconnu, ce corps qu'elle a oublié, parfois aussi, affamé pas le temps de se nourir, pas le temps de se reposer

$<<$ Il lui rappelle cruellement qu'il existe.

Sarah n'est plus qu'une ombre, un ersatz d'elle - même, un pâle reflet de celle qu'elle a été, que lui renvoie le miroir, sans pitié.>> (145)

Actuellement, Sarah perd ses cheveux par manettes. Ses cheveux, plus que toute autre chose, la désespère. L'oncologue l'avait averti en avance qu'à la deuxième séance de chimiothérapie, ils commenceront à tomber.

$<<$ (...) Une femme chauve, c'est une femme malade, peu importe qu'elle ait un pull magnifique, des talons hauts, un sac denier cri, personne ne les remarquera,(...) ce crâne nu (..) est un aveu, une confession, une souffrance. Un homme rasé peut - être sexy, une femme 
chauve sera toujours malade, pense Sarah. > (146)

La mère de Sarah balance ses réflexions et ses mémoires. Quand elle revient à la tête, elle se rappelle la résistance qu'elle avait à affronter, ce cancer qui l'attaquait en la dévorant.

< Même affaiblie, sa mère ne sortait jamais sans être maquillée, coiffée, les ongles faits. Les ongles, c'était un détail d'importance, (...) Pour beaucoup, ce n'était rien, une coquetterie, une futilité, mais pour elle c'était un signe, un geste qui signifiait : je prends encore le temps de m'occuper de moi. $>{ }^{(147)}$ )

Devant le miroir Sarah regarde ses ongles abîmés, ses cheveux épars. Elle perçoit comme si une minuscule partie de son être renonçait à se laisser discriminer. Sarah doit rentrer au combat, continuer la lutte. Au nom de sa mère, de ses trois enfants qui ont besoin d'elle, elle doit persister.

$<<$ Rapidement, elle s'habille. Pour cacher ses cheveux, elle attrape un bonnet dans le placard-c'est un bonnet d'enfant (...)Elle a mis un manteau, par-dessus trois pulls qu'elle a superposés. (...) Elle sait exactement où aller.>> (148)

Étant cinéaste, le récit de Colombani est découpé comme un film, elle commence d'abord à raconter les débuts de l'histoire de Smita en Inde, puis l'histoire de Giulia en Sicile ensuite, l'histoire de Sarah à Canada et elle retourne par cet ordre à continuer l'histoire de chacune, étape par étape. 
Revenons à l'histoire du voyage de Smita et de sa fille. D'abord, elles doivent rejoindre la grande route. Smita y a caché son vélo, dans un buisson ainsi qu'un petit emballage en carton contenant des nourritures. Smita et Lalita auront maints kilomètres à parcourir, avant d'atteindre la National Highway 56, reliant Lucknow à Varanasi où elles prendront un bus.

Smita déblaye les branches recouvrant son vélo. Il existe toujours. Mais il ne reste de l'emballage qu'elle avait préparé, qu'une bouteille d'eau

Smita place Lalita sur le porte-bagages et monte sur son vélo. Elle tremble en se cramponnant au guidon pour ne plus tomber. Assises au bord de la route, elles attendent le car deux heures. Enfin, il paraît une foule nombreuse se précipite vers ses portes. Il est trop difficile d'y monter. Elle détecte une demi-place pour elles deux, tout au fond, sur la banquette arrière.

Smita a essayé maintenant de reprendre ce chemin en sens inverse pour récupérer son vélo qu' elle a laissé dehors. Lalita se met à clamer en contrôlant le vélo à travers la vitre : un homme est monté dessus et s'éloigne à grande vitesse. Smita veut se jeter après lui mais elle risque de voir le car partir sans elle.

$$
\begin{aligned}
& <<\text { la mort dans l'âme, en regardant } \\
& \text { disparaître le morceau de ferraille usé } \\
& \text { qu'elle avait acheté jadis, et comptait } \\
& \text { revendre pour manger.>> (149) }
\end{aligned}
$$

Puis avec trois heures de retard, le car arrive enfin à la gare routière de Varanasi. Smita et sa fille sont fascinées par la dynamique de la ville. Des bus, des voitures, des camions

(La discrimination et ses formes ...) Dr. Abir Chéhata 
chargés de pèlerins se précipitent en direction du Gange et du Temple d'Or. La gare ferroviaire est au centre de la ville loin du lieu où le car les a quittées.

En s'orientant vers la gare ferroviaire, Lalita marche lentement afin de regarder des vues toutes nouvelles pour elle telles que les vitrines des magasins ainsi que les différentes marchandises qui se vendent au marché.

Lalita exhibe des signes d'épuisement, elles ont voyagé toute la journée, le ventre vide. Le plus difficile les attend encore, Smita le connaît.

La nuit est tombée lorsqu'elle parvient finalement au guichet.L'employé des chemins de fer révèle un air consterné quand elle demande deux billets pour Chennai, le jour-même. Les billets se réservent plusieurs jours en avance, riposte-t-il, à la dernière minute, les trains sont souvent complets. Smita insiste de partir, maintenant, au plus vite possible. Elle donne à l'employé quelques roupies mises pour leur repas. Celui-ci revient avec deux tickets de $\langle$ Sleeper class $>$, la classe la moins chère, dans le train du lendemain

$<$ Par la suite, Smita apprendra [qu'il]
(...) n'existe pas de restriction sur le
nombre de voyageurs par wagon dans
cette classe, (...) L'employé a joué de sa
crédulité pour lui soutirer quelques
roupies, elle le comprendra trop tard.>>
$(150)$

Finalement Smita et Lalita trouvent le train. Elles montent dans le wagon bleu de < sleeper class>, une voiture sans air conditionné, au confort usé, désuet, où foisonnent $<<$ blattes et souris $\gg$. $^{(151)}$

(La discrimination et ses formes ...) Dr. Abir Chéhata 
Les deux ripent difficilement dans le compartiment amassé, jusqu'à une petite place sur une banquette en bois . $<$ Leur train est un omnibus, moins cher que l'express. Il s'arrête partout et roule lentement. (...) C'est l'humanité tout entière qui voyage ici, (..)suffocante, épuisée dans ces wagons de dernière classe. >> (152)

En plus,

<<une odeur pestilentielle envahit le
wagon, une puanteur d'urine et
d'excréments mêlés. C'est ainsi à chaque
gare que le train dessert-les habitants des
villes ont l'habitude de venir se soulager
près des voies de chemins de fer.>> (153)

À côté de Smita, une grosse femme raconte qu'elle se dirige vers le temple de Tirupati pour y présenter une offrande - son fils est tombe malade. Un guérisseur lui a conseillé de faire un sacrifice dans un temple, et son fils a guéri. Aujourd'hui, elle va remercier Vishnou pour ce miracle en mettant des fleurs au pieds de sa statue. Smita réfléchit à la promesse déjà faite à Vishnou avant d'effectuer ce voyage où $<<$ Elle doit tenir parole, se dit-elle.>> (154)

Alors, elle décide de ne plus suivre leur route jusqu' à Chennai et lorsque le train s'arrêtera en gare de Tirupati, elles en descendront pour gagner la montagne sacrée afin de rendre hommage à leur divinité. Le train s'arrête en gare de Tirupati Beaucoup de pèlerins se déversent sur les quais lestés de bagages, de nourritures, de fleurs. Tous se dirigent à la sortie, en direction de la colline sacrée.

Le vrai pèlerinage se fait à pied. Smita n'est pas venu de très loin pour se lâcher à la facilité. Elle enlève ses sandales et

(La discrimination et ses formes ...) Dr. Abir Chéhata 
celles de Lalita comme le désire la tradition, en symbole d'humilité, pour commencer la montée des escaliers aux portes du temple.

Au premier quart du trajet, la fillette n'en peut guère alors, Smita soulève le petit corps faible de Lalita sur son dos pour poursuivre la montée.

Quand Smita termine sa montée, elle s'assoit pour reprendre son souffle devant les portes du temple

$<$ Tirumala est un monde en lui-même, plus peuplé qu'une ville. (...) On y accède en achetant un ticket.>>(155)

A l'aube, Smita et sa fille Lalita ont soudé les pèlerins devant l'entrée du temple. Par le goût sucré des laddus, pâtisseries présentées aux pèlerins, Smita se sent prête à tous les sacrifices. Elle n'a plus encore dit à Lalita ce qui les attend Si les plus riches posent des offrandes de nourritures, de fleurs, et de bijoux, les plus pauvres présentent l'unique bien qu'ils possèdent: leurs cheveux.

$$
\begin{aligned}
& <<(. .) \text { faire don de ses cheveux, c'est } \\
& \text { renoncer à toute forme d'ego, accepter de } \\
& \text { se présenter à Dieu dans son apparence } \\
& \text { la plus humble, la plus nue.>> (156) }
\end{aligned}
$$

Après s'être enfoncées dans le temple, Smita et Lalita se lancent entre les couloirs où des milliers de Dalits attendent pendant des jours. Or, cette attente s'allonge jusqu'à 48 heures. Leur tour est venu. Le barbier fait signe à Smita de marcher. Celle-ci obéisse, avec dévouement. Elle s'agenouille, ferme les yeux en commençant à réciter une prière à voix basse

$$
\begin{aligned}
& <<(. .) \text { Lorsqu'[elle] rouvre les yeux, son } \\
& \text { crâne est lisse comme un æuf. (...)C'est }
\end{aligned}
$$


une sensation nouvelle, (..) Maintenant son âme et son corps sont purs. Elle se sent apaisée. Bénie. Protégée. >>(157)

À son tour, Lalita s'avance devant le barbier en tremblant. Smita murmure avec elle la prière qu'elles ont plusieurs fois récitée devant le petit autel à Badlapur.

$<(.$.$) Sans cheveux, leurs visages se$ ressemblent plus qu'avant, plus que jamais. (..)Elles se tiennent par la main, et se sourient. Elles sont arrivées jusquelà. Le miracle s'est accompli. (..)Demain, une nouvelle vie commence.>> (158)

Dans le récit de Giulia, Kamal essaye d'expliquer la solution proposée de sa part pour sauver l'atelier des perruques en disant que s'il est interd it aux sikhs de se couper les cheveux, il n'en est pas le même cas pour les hindous dans son pays. Ceux-ci les coupent, par contre, par milliers pour les offrir à leurs divinités dans les temples

$\ll(.$.$) Si la matière première vient à$ manquer ici, conclut-il, il faut aller la chercher là-bas. Importer. C'est la seule façon de sauver l'atelier.>> (159)

Le mot " Importer" paraît à Giulia à peu près étrange. Cette idée lui plaît mais après une seconde, elle lui semble insensé. Kamal assure qu'il peut l'aider, il - sera un trait - d'union entre l'Inde et l'Italie. Revenant chez elle, Giulia allume son ordinateur passant la nuit à faire des recherches. Sur Internet, elle voit des images d'indiennes et d'indiens qui viennent dans les temples offrir leurs cheveux à leurs divinités espérant une meilleure récolte ou un mariage heureux ou bien une bonne santé. Giulia trouve aussi un homme d'affaires

(La discrimination et ses formes ...) Dr. Abir Chéhata 
anglais, dans cet article qu'elle vient de détecter ;faisant fortune dans le commerce des cheveux importés Dans son usine, près de Rome, d'abord, il provient les mèches indiennes par tonnes.

$<<$ La marchandise arrive par avion à l'aéroport de Fiumicino avant d'être acheminée dans une zone industrielle au nord de la ville, où elle est traitée dans d'immenses ateliers. >> (160)

Puis il éclaire comment les cheveux sont traités, trempés dans des bains de <dépigmentation > ${ }^{(161)}$ avant d'être teintés en blond, châtain ou roux devenant ainsi pareils aux cheveux européens. Ensuite, ces cheveux sont classés, assemblés en paquet et envoyés aux quatre recoins du monde où ils sont modifiés en extensions ou en perruques.

Finalement,son usine se transforme en multinationale comptant aujourd'hui des endroits de production sur trois continents en assurant $80 \%$ du marché.

Giulia est embarrassée, inquiète se demandant

$<<$ (...) Qui est-elle pour se croire à la hauteur de ce projet? (..)S'il a réussi, ne le peut-elle aussi ?>> (162)

Elle énonce que la réelle trahison réside de renoncer à ce projet, d'annuler le travail de trois générations. Brusquement, Giulia décide de ne pas épouser Gino Battagliola, ni de laisser l'atelier fermé.

$<$ L'idée de Kamal est un don, une chance, une providence. (...) elle ne l'a pas rencontré par hasard (...) Il lui a été 
envoyé. (...) Il est là le signe, le miracle qu'elle attendait. >> (163)

Dans le salon de la maison familiale, Giulia expose son projet à sa mère et à ses sœurs. Les jours précédents, elle a travaillé durement en faisant une étude de marché et en préparant un dossier pour la banque.

< relle se sent investie d'une mission quasi divine. Elle ignore d'où lui vient cette confiance, cette énergie soudaine. Est-ce la présence bienveillante de Kamal à ses côtés? Est-ce son père, du fond de son coma, qui lui donne sa force et sa foi? >> (164)

La mamma refuse en disant que les Lanfredi s'approvisionnent en Sicile, la cascatura représente une coutume ancestrale. On ne peut pas donc bouleverser la tradition. Francesca participe à la controverse en étant d'accord avec la mamma. Quant à Adela, celle-ci ne prononce aucun mot.

$<<$ La discussion vire à l'affrontement $>>$ (165)

Sans l'acceptation de sa mère et ses sœurs, Giulia ne pourra rien faire. Son enthousiasme s'effondre cédant la place à l'hésitation et à l' inquiétude.

Giulia se dirige à l'hôpital essayant de trouver refuge au chevet de son père, lui disant son ennui pour l'atelier. Ce jour, avant de mourir, le papa serre sa main comme pour lui dire adieu, comme pour lui dire encore : Vas-y

$<<$ Il lui a passé le relais, avant de s'en aller.>> (166)

Pour sortir de l'impasse, Giulia propose d'organiser un vote pour les ouvrières, le sujet les concerne aussi : Celles-ci vont

(La discrimination et ses formes ...) Dr. Abir Chéhata 
choisir entre deux solutions :la première, une nouvelle orientation de l'atelier exigeant l'importation de cheveux indiens. La deuxième, la fermeture en plus un licenciement discuté.

$$
\begin{aligned}
& \text { <Bien sûr, la première solution } \\
& \text { comporte des risques, des aléas certains } \\
& \text { que Giulia ne leur a pas caché.>> (167) }
\end{aligned}
$$

La mère et ses trois filles sont présentes dans la grande salle de l'atelier. D'une main apeurée, Giulia ouvre chaque papier jeté dans le chapeau du père comme un dernier hommage.

$$
\begin{aligned}
& <\text { Par sept voix contre trois, la majorité } \\
& \text { a tranché. }>>(168)
\end{aligned}
$$

Par le moyen de Kamal, Giulia forme un contact en Inde avec un homme installé à Chennai. Celui-ci parcourt le pays ainsi que ses temples à la recherche de cheveux à acheter.

Kamal se trouve souvent près de Giulia. Il s'intéresse à l'accompagner à l'aéroport. Dans l'entrepôt, elle signe un reçu signalant sa possession de la marchandise. D'une main tremblante, elle saisit un cutter pour fendre largement le colis.

$<<$ (...) des cheveux longs, très longs, noir de jais, (...) Juste à côté, une autre mèche : un peu moins longue, (...) Ils ont été achetés le mois dernier au temple de Tirupati, (..) le lieu de culte le plus fréquenté au monde, ... >> (169)

À cette information, Giulia pense à ces hommes et ces femmes inconnues pour elle, qui viennent faire don de leurs cheveux. Tel est le cas de Smita et sa fille Lalita, leurs cheveux ont fait un très long voyage de l'Inde à Sicile pour finir entre les mains de Sara à Canada. 
De même,

$<<$ Ils ne saurant jamais où sont partis leurs cheveux, quel périple incroyable ils auront accompli, quelle odyssée. (...) Un jour quelqu'un, quelque part dans le monde, portera ces mèches que ses ouvrières vont démêler, laver et traiter. Cette personne ne se doutera pas du combat qu'il aura fallu mener. $>>$ (170)

En se dirigeant vers le salon de « chevelures d'appoint ${ }^{(171)}$

Où Sarah a choisi un modèle, en cheveux humains. Sarah admire la perruque d'un air étonnant :ces cheveux sont de la même que les siens. Sarah observe dans le miroir: une femme nouvelle se tient en face d'elle, qui lui est identique et qui est en même temps une autre. Cette chevelure le lui redonne son énergie, sa retenue et sa fermeté, tout ce qui réalise qu' elle est puissante, solide, vaniteuse et magnifique.

Sarah veut sortir vainqueur de cette lutte, épuisée peut être mais debout. Quoi qu'il en soit le nombre de jours, de semaines, de mois, d'années de traitement, elle vouera toute sa force à lutter corps et âme contre le cancer.

$<<$ (...) En attendant, il faut lutter contre la mandarine, avec les armes que la nature a bien voulu lui donner : son courage, sa force, sa détermination, son intelligence aussi. Sa famille, ses enfants, ses amis.>> (172)

À vrai dire, nous constatons que Colombani a su relier par excellence les trois espaces à travers les cheveux tressés qui ont voyagé du temple de Tirupati en Inde à l'aéroport de 
Sicile pour arriver enfin dans un magasin de perruques à Montréal au Canada.

Ainsi, la romancière nous fait part de son écriture tressée en raccordant le fil de trois récits de femmes dans trois coins différents du monde.

\section{Conclusion :}

Dans la tresse, Colombani nous confie un récit qui se divise en de courts chapitres retenant ainsi les lecteurs qui désirent en savoir plus à la fin de chaque partie.

La romancière raconte l'histoire de trois femmes chacune à leur tour en les plaçant face à de dures épreuves : Smita a été victime d'une discrimination raciale, Giulia a été proie à une discrimination familiale quant à Sarah, elle a subi sa part de discrimination à cause de sa déficience.

Colombani veut bien appuyer sur les évènements marquants dans chaque histoire. De même, elle désire avouer son point de vue contre la discrimination car chaque évènement se rapporte à une des conséquences négatives de la société. Chacune de ces trois héroïnes a son niveau et a sa manière d'affronter plusieurs défis liés à sa condition féministe dont les droits ne sont plus acquis....

La romancière nous prouve que malgré qui nous sommes, d'où nous venons, que nous sommes riches ou pauvres : un fait est commun à chaque être humain : l'espoir d'une vie meilleure. Ce n'est qu'un cri qui émane de l'âme des femmes pour une renaissance allégorique.

$<$ Les femmes dépeintes par Laetitia

Colombani ont cette pureté de l'âme qui

(La discrimination et ses formes ...) Dr. Abir Chéhata 
En outre,

les conduit à ne pas céder à la facilité des coups-bas. ou de la méchanceté. Et à chaque fois, qu'elles tombent, elles puisent une force encore plus viscérale pour se relever. >>

$<<$ 'expression $\quad<<$ discrimination positive>>, a laquelle le Grand Dictionnaire terminologique québequois préfère les appellations $<$ action positive >>ou <<dédiscrimination>> vise à corriger, réparer, inverser la discrimination, en agissant sur les groupes qui sont habituellement défavorisés. En effet, les politiques et le droit peinent à rétablir l'équilibre entre les groupes sociaux, car les groupes défavorisés ne bénéficient pas des mêmes armes (...) que les autres. Ils sont donc objectivement désavantagés, quand bien même aucune discrimination ne serait à l'ouvre.>> (1)

L'intrigue bien peignée d'histoires de cheveux qui chemine de l'une à l'autre en se formant en une tresse. Avec le concept de la tresse, Colombani découvre le juste équilibre pour tisser sa trame.

Ce roman émouvant, fascinant, inoubliable, est marqué par une fluidité d'écriture directe, géniale ainsi qu'un thème très important surtout la condition inhumaine imposée aux femmes Intouchables à l'Inde qui nous émeut et nous secoue.

\section{1) https://fr.wikipedia.org/wiki/Discrimination/25-11-2020}

(La discrimination et ses formes ...) Dr. Abir Chéhata 
Aussi, le style se met au service du récit et paraît s'accoutumer au rythme de la vie des personnages.

La tresse est un roman dédié <aux femmes courageuses〉> qui, grâce à leur patience, leur force se battent chaque jour pour obtenir la liberté et la dignité.

Ce roman permet de donner à toutes les femmes du monde le courage de repousser ce que leur dictent les rites de la famille, de la société, les coutumes et de frayer un pas en avant vers un avenir meilleur digne d'elles. 
1) https ://www.onlalu.com/livres/poches/la-tresse-laetitia-colombani27188

2) Colombani(L), La tresse, Éditions Grasset \& Fasquelle, 2017, p.11

3) Genette (G), Figures II, Paris, Seuil,1969, p.49

4) Ibid., p.56

5 ) https ://www.babelio.com/auteur/laetitia-

Colombani/433135/bibliographie

$6 \quad$ http: Mittexpress.iut.u-bordeauxmontaigne.fr/index.php/2019/02/19/laetitia-colombani-la-tresse-2

7) https : I/fr.wikipedia.org/wiki/Discrimination/25/11/2020

8) Cf Colombani (L), La tresse, op.cit., pp.159-160

9) Ibid., p.15

10) Grignon $(C),<<$ Racisme et ethnocentrisme de classe >>, Bulletin interne de l'Association critiques sociales, no.2, décembre1988, p. 5

11) Idem.

12 ) https: //www.filsantejeunes.com/la-discrimination-c est-quoi6375/29-11-2020

13) Colombani (L), La tresse, op.cit., p.18

14) Ibid., p.16

15) Ibid., p.18

16) $\underline{\text { Idem }}$

17) Colombani (L), La tresse, op.cit., p.43

18) Ibid., p.44

19 ) https:// www.babelio.com lauteur/laetitiacolombani/433135/bibliographie

$20)$ Grignon (C), art. cit, p.2 nous suivons Grignon dans cette caractérisation générale du racisme depuis une dizaine d'années : < le racisme est déni d'humanité, et non pas déni d'identité.>>

21) Colombani (L), La tresse, op.cit., p17

22) Idem

23) Ibid., p.15

24 Jouve(V), L'Effet - Personnage dans le roman, Presses universitaires de France, collection $<<$ Écriture $>, 1992$ (2 -ème édition 1998) pp.111-112

25)Colombani (L), La tresse, op.cit., p.19

26) Ibid., p.47

27) Ibid., p.66

28) Ibid., p.68

(La discrimination et ses formes ...) Dr. Abir Chéhata 
29) Idem

30) Taguieff (Pierre - André), Face au racisme, Tome II, éditions La Découverte, Paris, 1991, p.28

31) https://www.filantejeunes.com/la-discrimination-cest-quoi6375/29-11-2020

32) https://www.humanrights.ch/fr/pfi/droits-

humains/discrimination/dossier-non- discrimination /qu-est-ce-quela-discrimination/29-11-2020

33) Colombani (L), La tresse, op.cit., pp.70-71

34) https://www.humanrights.ch/fr/pfi/droits-humains/discrimination/ dossier-non-discrimination/qu-est-ce-que-la-discrimination/29-112020

35) Taguieff (Pierre -André), Face au racisme, Tome II, op.cit. p.17

36) Colombani (L), La tresse, op.cit.., p.71

37) Ibid., p119

38) Ibid., p.72

39) Ibid., p.88

40) Hamon (PH), Le Personnel du roman système des personnages dans les Rougon-Macquart d'Emile Zola-Genève-Droz,1983, p.237

41) Colombani( $(L)$, La tresse, op.cit., p.121

42) Gérard $(G)$, Nouveau discours du récit, Paris, Seuil, coll. <<

Poétique >>, 1983, p.106

43) Colombani (L), La tresse, op.cit., p.71

44) Idem

45) https://www.amnesty.ch/fr/themes/discriminations/faits-chiffres-etnotions-de-base/definition-quest -ce-que-la-discrimination/29-11-2020

46) Colombani (L), La tresse, op.cit., pp.90 -91

47) https : //fr.wikipedia.org/wiki/Discrimination/25-11-2020

48) Taguieff (Pierre - André) Face au racisme, Tome I, op.cit., p.71

49) Colombani(L), La tresse, op.cit., p.92

50) https : I/fr.wikipedia.org/wiki/Discrimination/25-11-2020

51) Colombani(L), La tresse, op.cit., p.117

52) Reuter (Yves), Introduction à l'analyse du roman, Paris, Armand Colin, 2013, p.40-41

53) Colombani(L), La tresse, op.cit., p.25

54) Chevalier, Jean et GHEERBRANT, Alain, Dictionnaire des symboles, Paris, Robert Laffont.S .A et Jupiter1982 pp.740-741

(La discrimination et ses formes ...) Dr. Abir Chéhata 
55) Colombani (L), La tresse, op.cit., p.23

56) $\underline{\text { Idem }}$

57) Colombani( $(L) \underline{\text { La tresse, op.cit., p.26 }}$

58) Ibid, p.50

59) $\underline{\text { Idem }}$

60) Ibid, p.51

61) $\underline{\mathrm{Ibid}}$, p.53

62) $\underline{\text { Ibid. }}$., p.54

63) Ibid., p.74

64) $\underline{\text { Ibid., }}$ p.75

65) Ibid., p.29

66) Ibid., p.77

67) $\underline{\text { Idem }}$

68) Ibid., p.79

69) Ibid., p.80

70) $\underline{\text { Idem }}$

71) Ibid., p.98

72) $\underline{\text { Ibid. }}$., p.122

73) Ibid., p.123

74) $\underline{\text { Idem }}$

75) Ibid., p.26

76) Ibid., p. 24

77) Ibid., p. 125

78) Tomachevski(B), Thématique trad Franc T. Todorov, théorie de la littérature, Paris Seuil coll < tel que >> 1965, p. 295

79) Colombani (L)La tresse, op.cit., p.149

80) Ibid., p.151

81) Ibid., p.153

82) $\underline{\text { Ibid. }}$., p.155

83) $\underline{\text { Ibid., }}$ p.174

84) Ibid., p.33

85) $\underline{\text { Idem. }}$.,

86) Idem.,

87) $\underline{\text { Ibid., }}$ p. 35

88) https:// www.babelio .com/auteur/laetitiacolombani/433135/bibliographie

89) Colombani (L) La tresse, op.cit., p.37

90) $\underline{\text { Idem }}$

(La discrimination et ses formes ...) Dr. Abir Chéhata 
91) Ibid., p.34

92) $\underline{\text { Ibid. }}$, p.32

93) $\operatorname{Erman}(M)$, Poétique du personnage du roman,Ellipses,Paris,2006,pp.9-10

94) MERLE , PIERRE $<<$ Classes Sociales $>><$ Groupes de statut $><<$ Partis $>$ Une Analyse Systémique des Stratifications Sociales Wébériennes >> in Ressources en Sciences Économiques et Sociales SES - ENS( Cours en ligne),p.09,

mis en ligne le 28 février 2015, consulté le 25 mai 2019.

URL :http://ses.ens-lyon.fr/ses/fichiers/pierre-merle-weber-

stratification-fev-20151425210929771.pdf

95) Colombani(L)la tresse, op.cit., p.55

96) Ibid., p.58

97) Ibid., p.59

98) Idem

99) $\underline{\mathrm{Ibid}}$., $p .60$

100) Idem

101) Idem

102) $\underline{\text { Idem }}$

103) $\underline{\text { Ibid. }}$, p.83

104) Ibid., p.85

105) Idem

106) Ibid., p.86

107) Ibid., p.106

108) Ibid., p.107

109) Ibid., p.109

110) Idem

111) $\underline{\text { Ibid. ., p.111 }}$

112) $\underline{\text { Idem }}$

113) $\underline{\text { bid }}$. .p. 112

114) $\underline{\text { Ibid. }}$, p.113

115) Ibid., p. 128

116) Ibid., p.129

117) $\underline{\text { Idem }}$

118) Ibid., p. 130

119) I Idem

120) Ibid., p.131

(La discrimination et ses formes ...) Dr. Abir Chéhata 


\section{1) $\underline{\text { Idem }}$}

122) $\underline{\text { Idem }}$

123) Ibid., p.133

124) Ibid., p.134

125) $\underline{\text { Idem }}$

126) Ibid., p.158

127) Ibid..p.159

128) $\underline{\text { Idem }}$

129)Ibid.,p.162

130)Ibid.,p.163

131)Ibid.,p.164

132)_VANDENBERGHE, Frédéric, $<<$ La Notion de Réification .

Réification Sociale et Chosification Méthodologique >> , in L' Homme et la Société [ Enligne ], 1992 , no 103, Aliénations nationales ,pp.81 82 , mis en ligne le 25 septembre 2015 , consulté le 25 mai 2019. URL : http// www. persee . fr/ doc/homso-00184306-1992- num-103-1-2615 133)CHARBONNIER, Vincent,$<<$ La Réification chez Lukács : ( la madeleine et les cendres) >> [ En ligne ], in CHANSON, Vincent,CUKIER,Alexis et MONTFERRAND, Frédéric (dir.), La Réification :Histoire et Actualité d' un Concept Critique , Paris , la Dispute , 2014, p.44, mis en ligne le 20 février 2014, consulté le 25 mai 2019. URL : https // hal-ens-lyon.archives ouvertes. fr / ensl- 00762337v 7

134) VANDENBERGHE , Fréderic ,op.cit, p.85

135) Colombani (L), La tresse , op.cit. , p.31

136) Idem

137) CHARBONNIER , Vincent, op.cit. , pp.43-44

138) Colombani (L), La tresse , op.cit. , p.164

139) Ibid.,p.165

140) Ibid.,pp.166-184

141) Ibid.,p. 186

142) $\underline{\text { Ibid. } ., p 187}$

143) Ibid.,pp188-189

144) $\underline{\text { Idem }}$

145)Ibid.,p.191 
146) $\underline{\text { Idem }}$

147)Ibid.,p.192

148) Ibid.,p.193

149) Ibid.,p.138

150) Colombani (L), La tresse, op.cit. , p.144

151) Ibid.,p.168

152) Ibid..pp.168-169

153) Ibid p.170

154) Ibid p. 172

155) Ibid p.182

156) Ibid., p. 202

157) Ibid., p.204

158) Ibid., p. 205

159) Ibid., p.174

160) Ibid., p.176

161) $\underline{\text { Idem }}$

162) Ibid., p.177

163) $\underline{\text { Idem }}$

164) Ibid., pp.194-195

165) Ibid., p.198

166) Ibid., p.206

167) Ibid., p.208

168) $\underline{\text { Idem }}$

169) Ibid., p.210

170) $\underline{\text { Idem }}$

171) Ibid., p.213

172) Ibid., p. 219 


\section{Bibliographie :}

\section{I-Corpus :}

- Laetitia(Colombani), La tresse, Grasset \& Fasquelle, 2017

\section{II-Ouvrages Généraux :}

- Genette, (G), Figures II, Paris, Seuil, 1969.

Nouveau discours du Récit, Paris, Seuil,col «Poétique »,1983.

- Hamon, (P), Le Personnel du roman système des personnages dans les

Rougon -Macquart d'Emile Zola -Genève -Droz,1983.

- Reuter, (Y), Introduction à l'analyse du roman, Paris, Armand Colin,2013.

- Taguieff (Pierre -André), Face au racisme, Tome I, Tome II Éditions La Découverte, Paris,1991.

- Jouve, (V) ,L'Effet-Personnage dans le roman, PUF, collection <écriture> 1992 (2-ème édition 1998).

- Tomachevski (B), Thématique, trad. franc T.Todorov, théorie de la littérature, Paris, Seuil, coll «Tel que» 1965. 


\section{III-Articles :}

- CHARBONNIER, Vincent,$\ll$ La Réification chez Lukács : ( la madeleine et les cendres) >> [ En ligne ], in CHANSON, Vincent, CUKIER, Alexis et MONTFERRAND, Frédéric (dir.), La Réification :Histoire et Actualité d' un Concept Critique, Paris, la Dispute, 2014 , p.44, mis en ligne le 20 février 2014 , consulté le 25 mai 2019 . URL : https//hal-ens-lyon.archives -ouvertes.fr/ensl$00762337 \mathrm{v} 7$

- Grigon,Claude $<<$ Racisme et ethnocentrisme de classe >>,Bulletin interne de l'Association critiques sociales, no 2 , décembre 1988.

- VANDENBERGHE, Frédéric, $<<$ La Notion de Réification . Réification Sociale et Chosification Méthodologique >> in L' Homme et la Société [ Enligne ], $1992, \mathrm{n}^{\circ} 103$, Aliénations nationales ,pp.81 -82 , mis en ligne le 25 septembre 2015 , consulté le 25 mai 2019. URL : http// www. persee . fr / doc/homso00184306-1992- num-103-1-2615

\section{IV-Thèses :}

- Nawel(Salem), L'écriture tressée et l'image Kaléidoscopique de la mondialisation dans La Tresse de Laetiti Colombani Université Mohamed Lamine Debaghine-sétif 2, 2018/2019 


\section{$\underline{\text { V-Dictionnaires : }}$}

- CHEVALIER,Jean

et

GHEERBRANT,

Alain,Dictionnaire des symboles, Paris , Robert Laffont S.A. et Jupiter, 1982.

\section{VI-Sitographie :}

- https://www.onlalu.com/livres/poches/la-tresselaetitia-colombani-27188

- https://www.babelio.com/auteur/laetitiacolombani/433135/bibliographie

- https://fr.wikipedia.org/wiki/Discrimination/25/11/202 0

- https://www.filsantejeunes.com/la-discrimination-c est-quoi-6375/29-11-2020

- https://www.humanrights.ch/fr/pfi/droits- humains/ discrimination/dossier-non-discrimination/qu - estce-que - la-discrimination/29-11-2020

- https://www.amnesty.ch/fr/themes/discriminations/faits -chiffres-et-notions-de-baseldefinition-quest -ce-quela-discrimination/29-11-2020 


\section{التمييز و أشكاله في "الجديلة" للكاتبة كولومباني}

تروي كولومباني في قصتها "الجديلة" قصة ثلاث سيدات، ثلاث حيوات في ثلاثة

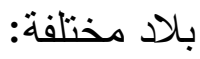

الأولى في الهند حيث تعيش "سميتا" إمرأة منبوذة بائسة في قرية بدلابور، منزوجة من صائد للفئران تقوم بمهام معيبة تتمثل في تنظيف مراحض الطبقات الأكثز نبالة حالها كحال طبقة المنبونين، الطبقة الأكثز فقرا في قريتها. شئ

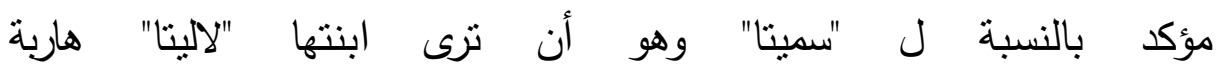
من هذه الحالة الرثائية بدخولها المدرسة. الثانية في إيطاليا حيث تعيش "جيليا" و هي تعمل في مشغل لصناعة الثعر المستعار المدار من قبل والدها. يدخل والدها في غيبوبة عميقة ضحية لحادث وتكتشف "جيليا" فجأة أن المنشأة الأسرية على وجه الإفلاس و هذا يتطلب إذا إيجاد حل لإنقاذ هذا الوضع.

والثالثة في كندا حيث تعيش "سارة" محامية شهورة و لديها ثلاثة أطفال. تترقى "سارة" لتصل إلى القمة في مكتب المحاماة عندما يخبرها الطبيب أنها مصابة بمرض السرطان.

تريد الكاتبة كولومباني أن تصر جيدا على الأحداث المهمة في كل حياة ـ فهي ترغب أيضا أن تصرح بوجهة نظرها ضد التمبيز لأن كل عنصر بتعلق بإحدى

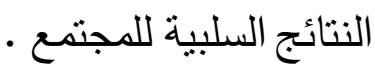

